



\title{
Photometric variability in the Kepler field
}

\author{
Dissertation \\ zur Erlangung des mathematisch-naturwissenschaftlichen Doktorgrades \\ "Doctor rerum naturalium" \\ der Georg-August-Universität Göttingen \\ im Promotionsprogramm ProPhys \\ der Georg-August University School of Science (GAUSS)
}

vorgelegt von

Timo Reinhold

aus

Bremerhaven

Göttingen, 2013 


\section{Betreuungsausschuss}

Prof. Dr. Ansgar Reiners, Stellare Astrophysik, Institut für Astrophysik Göttingen (IAG)

Prof. Dr. Stefan Dreizler, Stellare Astrophysik, IAG

Dr. Jose Fernandez, Stellare Astrophysik, left IAG Feb. 2011

Mitglieder der Prüfungskommission

Referent: Prof. Dr. Ansgar Reiners, Stellare Astrophysik, IAG

Korreferent: Prof. Dr. Stefan Dreizler, Stellare Astrophysik, IAG

Weitere Mitglieder der Prüfungskommission:

Prof. Dr. Wolfram Kollatschny, Extragalaktische Astrophysik, IAG

Prof. Dr. Wolfgang Glatzel, Computational Radiation Hydrodynamics, IAG

Prof. Dr. Hardi Peter, Solar and stellar astrophysics, MPS

Prof. Dr. Andreas Tilgner, Geophysikalische Fluiddynamik, Institut für Geophysik

Tag der mündlichen Prüfung: 


"Die Forderung, dass Auschwitz nicht noch einmal sei, ist die allererste an Erziehung. Sie geht so sehr jeglicher anderen voran, dass ich weder glaube, sie begründen zu müssen noch zu sollen."

Theodor Wiesengrund Adorno

Auszug aus Erziehung zur Mündigkeit 


\section{Contents}

List of Figures $\quad x$

List of Tables $\quad$ xi

Abstract xiii

Overview xv

\begin{tabular}{ll}
\hline I. Introduction & 1
\end{tabular}

1. Motivation 3

1.1. Rotation . . . . . . . . . . . . . . . . . . . . . . 3

1.2. Differential Rotation . . . . . . . . . . . . . . . . . . . . 3

1.3. Measurement techniques . . . . . . . . . . . . . . . . . . 4

1.4. High precision photometry: CoRoT \& Kepler . . . . . . . . . . . . . . . . 5

1.5. Goals and Challenges . . . . . . . . . . . . . . . . . . . 5 5

$\begin{array}{ll}\text { II. Simulations } & 9\end{array}$

$\begin{array}{ll}\text { 2. Simulations } & 11\end{array}$

2.1. The Model . . . . . . . . . . . . . . . . . . . . . . . . . . . . 11

2.2. Monte-Carlo simulation . . . . . . . . . . . . . . . . . . . 12

\begin{tabular}{ll}
\hline 3. Period determination & 14
\end{tabular}

3.1. Lomb-Scargle periodogram . . . . . . . . . . . . . . . . . . . . . . . 14

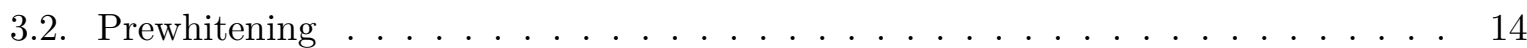

3.3. Period selection . . . . . . . . . . . . . . . . . . . . . . 15

3.4. Sample Properties . . . . . . . . . . . . . . . . . . . . 17

4. Simulation Results 20

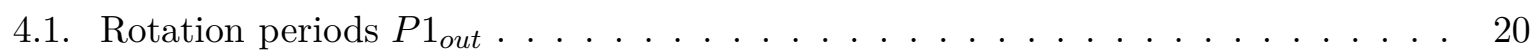

4.2. Differential Rotation . . . . . . . . . . . . . . . . . . . . . . 20

\begin{tabular}{|ll}
\hline III. Kepler data & 27
\end{tabular}

$\begin{array}{ll}\text { 5. Selection of active stars } & 29\end{array}$

6. Period determination II 31

6.1. Lomb-Scargle periodogram \& Pre-whitening . . . . . . . . . . . . . . . . . . . . 31

6.2. Period selection . . . . . . . . . . . . . . . . . . . . . 31 
7. Limits \& Examples 33

7.1. Zero crossings . . . . . . . . . . . . . . . . . . . . . . . 33

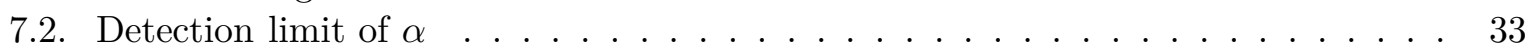

7.3. Examples: Light curves, Periodograms, and Rotation Periods . . . . . . . . . . . 34

8. Kepler Results 38

8.1. Rotation Periods . . . . . . . . . . . . . . . . . . . . . . 38

8.2. Rotational Braking . . . . . . . . . . . . . . . . . . . . 38

8.3. Relative Differential Rotation $\alpha$. . . . . . . . . . . . . . . . . . . . . 41

8.4. Absolute horizontal shear $\Delta \Omega \ldots \ldots \ldots \ldots \ldots$. . . . . . . . . . . . 43

8.5. False Positives . . . . . . . . . . . . . . . . . . . . . 44

8.6. Differential Rotation bevond $\alpha=0.3 \ldots \ldots \ldots \ldots \ldots$. . . . . . . . . . 47

\begin{tabular}{ll}
\hline IV. Discussion & 51
\end{tabular}

9. Comparison to other observations 53

$\begin{array}{ll}\text { 10. Comparison to theory } & 55\end{array}$

11. Summary \& Conclusions 56

$\begin{array}{ll}\text { 12. Outlook } & 58\end{array}$

\begin{tabular}{ll}
\hline Bibliography & 60
\end{tabular} 


\section{List of Figures}

1.1. SOHO/MDI image of a large sun spot group. . . . . . . . . . . . . . . . 4

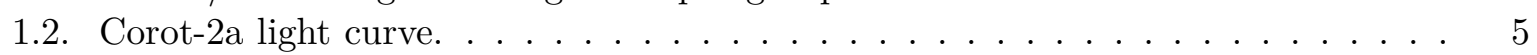

2.1. Simulated stellar surface and resulting light curve using MODSTAR. . . . . . . . 12

3.1. Simulated light curve with best global sine fit. . . . . . . . . . . . . . . . 15

3.2. Comparison of stellar parameters for the samples S2 and S1. . . . . . . . . . 18

4.1. Comparison of input and output rotation periods . . . . . . . . . . . . 21

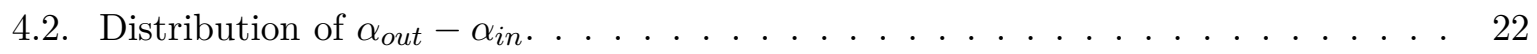

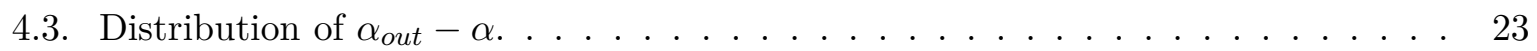

4.4. Distribution of the relative error $\left|\alpha_{\text {out }}-\alpha_{\text {in }}\right| / \alpha \ldots \ldots \ldots \ldots$. . . . . . . 24

5.1. Temperature vs. gravitv of all Kepler Q3 stars. . . . . . . . . . . . . . . . 29

5.2. Distribution of $R_{\text {nar }}$ for all Kepler Q3 stars. . . . . . . . . . . . . . . . . 30

7.1. Frequencv $f_{1}$ vs. number of zero crossings in Q3. . . . . . . . . . . . . . . . . 34

7.2. Light curve. global fit and periodograms of the star KIC 1995351. . . . . . . . . 36

7.3. Light curve, global fit, and periodograms of the star KIC 1869783. . . . . . . . . 37

8.1. Distribution of rotation periods $P_{1}$ and $P_{2} \ldots \ldots \ldots \ldots \ldots$

8.2. Rotation period $P_{1}$ against $B-V$ of 24.124 stars incorporating previous period measurements. 40

8.3. Densitv plot of rotation period $P_{1}$ vs. $R_{\text {nar }} \ldots \ldots \ldots \ldots \ldots \ldots$. . . . . 41

8.4. Age distribution of the active Kepler stars inferred from rotation periods $P_{1}$. . . 42

8.5. Rotation period $P_{\min }$ vs. $\alpha$ for all stars with 2 detected periods. . . . . . . . 43

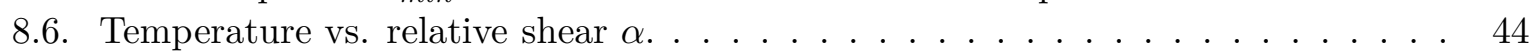

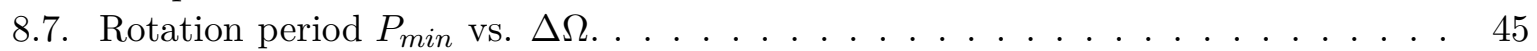

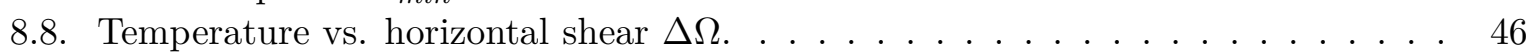

8.9. Densitv plot in the $T_{\text {eff }}-\alpha$ plane for different values of $\alpha_{\max }$. . . . . . . . . . 48

8.10. Density plot in the $P_{1}-\alpha$ plane for different values of $\alpha_{\max } . \ldots \ldots . . .49$ 


\section{List of Tables}

2.1. Stellar simulation parameters . . . . . . . . . . . . . . . . . . . . . . . . . . . . . . . . . .

2.2. Spot simulation parameters . . . . . . . . . . . . . . . . . . . 13

3.1. Period selection process using the example of the light curve from Fig. 3.1 . . 17

4.1. Comparison of input and output rotation periods. . . . . . . . . . . . . 20

4.2. Comparison of input and output shear $\alpha_{\text {in }}$ and $\alpha_{\text {out }}$. . . . . . . . . . . . . . 24

8.1. Number of stars with second period found for different $\alpha_{\max }$ values. . . . . . . . 47

9.1. Comparison of rotation periods with previous rotation measurements for Kepler. 54 



\section{Abstract}

The Kepler space telescope monitors more than 160.000 stars with an unprecedented precision providing the opportunity to study photometric variability of thousands of stars. Our major focus lies on the detection of Differential Rotation (DR) by measuring different periods from photometric data resulting from co-rotating spots on the stellar surface. We developed a method to extract the most significant periods from a given light curve: The periods are derived from Lomb-Scargle periodograms in a successive way by prewhitening of the light curve combined with a global sine fit. We associate these periods to active regions (e.g. star spots or spot groups) rotating in and out of view at different latitudes.

To understand the variety of different Kepler light curves we decided to simulate the rotation of spotted stellar surfaces. Thus, we ran a large Monte-Carlo simulation of spotted, differentially rotating stars to understand the nature of spot distributions on the stellar surface, the resulting light curves, and their periodograms covering a large fraction of the parameter space. We applied our method to the simulated light curves and found very good agreement between the spot periods from the simulation, and the periods returned by our method. Not only the most significant rotation periods could be recovered fairly well but also the relative latitudinal shear was detected with high accuracy. Our method yields surprisingly good results which motivated us to apply this promising tool to Kepler data.

We analyzed Quarter 3 Kepler data and derived rotation periods $P_{1}$ between 0.5 and 45 days for more than 24.000 active Kepler stars. The periods are consistent with previous rotation measurements and with the concept of magnetic braking. Among those, in more than 18.000 stars a second period $P_{2}$ has been detected which is interpreted as surface DR. From these two periods we calculated the absolute and relative surface shear $(\Delta \Omega$ and $\alpha=\Delta \Omega / \Omega$, resp.) and show how these quantities depend on rotation rate and effective temperature. We find that the relative shear $\alpha$ increases with rotation period, and slightly decreases with effective temperature. The absolute shear $\Delta \Omega$ slightly increases between $3500 \mathrm{~K}<T_{\text {eff }}<6000 \mathrm{~K}$. Above $6000 \mathrm{~K} \Delta \Omega$ shows much larger scatter, but a weak dependence on rotation period. Furthermore, we show that our results are consistent with major predictions from mean-field theory and seem to support these models.

In this work latitudinal differential rotation has been measured for the first time for more than 18.000 stars drawing a completely new picture of DR. Due to the large number of stars false positives have been estimated in a statistical way. To what extent our observations are affected by a selection bias is not fully explored. I will address this problem in my future work using the whole Kepler time coverage. 



\section{Overview}

This thesis is organized in four parts: The first one introduces the main topic of this thesis - the measurement of DR from photometric data - summarizing previous measurements, theoretical approaches, and its open questions. Part I and III are taken from my first publications: A fast and reliable method to measure stellar differential rotation from photometric data, accepted by A\&A, and Rotation and Differential Rotation of active Kepler stars, submitted to A\&A. More than $99 \%$ of the text has been written by myself with minor language editing by my supervisor Ansgar Reiners, and the co-author of the second publication, Gibor Basri. Part I describes our model to simulate light curves of (differentially) rotating stars, and motivates the parameter space of the Monte-Carlo simulation. In the following we describe the period detection using Lomb-Scargle periodograms in a prewhitening approach, the selection process of the periods, and simulation results. Part III contains the application of our method to Kepler data. We describe how active stars have been selected from the whole sample, and which measures and limits we imposed to exclude false positives. Our results on rotation and differential rotation build the main character of this part and the whole thesis. The final part contains the discussion of our results, and a prospect to future work. 

Part I.

Introduction 



\section{Motivation}

\subsection{Rotation}

The interplay of stellar rotation and convection is the origin of various stellar activity phenomena, e.g. star spots, faculae, plage, flares. etc. Since rotation can be measured fairly easy, either from long-term monitoring of star spots, or broadening of spectral lines, the rotation rate is a well-known quantity for thousands of stars. For main sequence stars the rotation rate strongly depends on the stellar age. Due to rotational braking stars loose angular momentum over the time and slow down. Skumanich (1972) empirically found the relation that the stars' rotational velocity is proportional to the inverse square root of its age: $v_{\text {rot }} \sim t^{-1 / 2}$. Barnes (2003) shows that this relation holds for open cluster and Mount Wilson stars, furthermore providing a color dependence of the rotation period. Irwin et al. (2011) measure rotation periods for stars with masses below $0.35 M_{\odot}$, finding some exceptionally fast rotators. These stars do not follow the color-period relation from Barnes (2003) but they can be explained by a radius-dependent braking efficiency (Reiners \& Mohanty 2012). Nowadays, a method called gyrochronology (Barnes 2007) is being developed using Skumanich's relation in the opposite way to infer stellar ages from the rotation rate. Moreover the rotation rate strongly correlates with CaII emission it can be used as a measure of stellar activity. A relation between these properties is often called age-rotation-activity relation (Covey et al. 2011).

\subsection{Differential Rotation}

Stellar rotation is by no means uniform. In early F-type stars a convection zone starts to form growing towards later spectral types. The Coriolis force acts on turbulences in the convection zone. Its back reaction redistributes angular momentum and changes the global rotation behavior leading to Differential Rotation (hereafter DR) of the surface. A detailed theoretical description can be found, e.g. in Kitchatinov (2005). On the Sun the equatorial region rotates faster than the poles, i.e. the angular velocity $\Omega$ depends on the latitude $\theta$. This is usually described by the equation

$$
\Omega(\theta)=\Omega_{e q}\left(1-\alpha \sin ^{2} \theta\right)
$$

with $\Omega_{e q}$ being the angular velocity at the equator, and $\alpha=0.2$ the relative horizontal shear. In general $\alpha>0$ is known as solar-like DR, $\alpha<0$ is called anti solar-like DR, and $\alpha=0$ supplies rigid body rotation. The absolute shear $\Delta \Omega$ between the equator and the pole is linked to $\alpha$ by the relation

$$
\Delta \Omega=\Omega_{e q}-\Omega_{\text {pole }}=\alpha \Omega_{e q} .
$$

DR is believed to be one major ingredient of the driving mechanism of magnetic field generation on the Sun. Assuming an initial poloidal magnetic field with frozen field lines DR winds up the lines transforming an initial poloidal field into a toroidal field ( $\Omega$-effect). The opposite effect which transforms the toroidal field back into a poloidal one is called $\alpha$-effect 11 resulting from surface convection. In contrast to the $\Omega$-effect the $\alpha$-effect is able to produce a toroidal field

\footnotetext{
${ }^{1}$ The term $\alpha$ in this case originates from mixing length theory and should not be confused with our definition of the relative horizontal shear in eq. (1.1).
} 


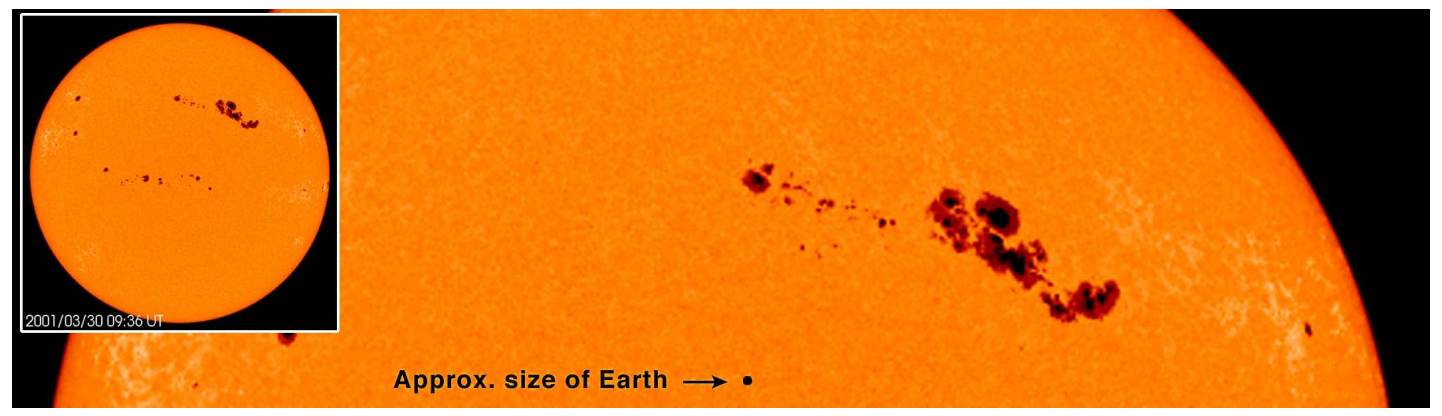

Figure 1.1.: SOHO/MDI image resolving a large sun spot groups and individual active regions.

from a poloidal one, and vice versa. The interplay of these phenomena is known as the solar $\alpha \Omega-$ dynamo which generates the magnetic field of the Sun and causes its 11 year activity cycle. There is evidence for different dynamo mechanisms in other stars. Turbulent dynamos produce strong magnetic fields and are able to transform poloidal into toroidal fields, and vice versa. Morin et al. (2008) showed that the M4 dwarf V374 Peg exhibits a strong magnetic field showing only weak signatures of DR. This effect becomes even more important when stars become fully convective (Morin et al. 2010). Furthermore, the strength of DR varies with spectral type. Barnes et al. (2005) found that $\Delta \Omega$ strongly increases with effective temperature. For temperatures above $6000 \mathrm{~K}$ this trend was confirmed by Reiners (2006). This could be a hint towards different dynamo mechanisms but the final role of DR is still not fully understood.

\subsection{Measurement techniques}

There are several ways to measure stellar rotation rates. The most common techniques are the long-term monitoring of active stars yielding rotation periods from star spots (Hall 1991), and the fit to individual spectral lines measuring rotational broadening in terms of $v \sin i$. Furthermore, line core variations in the CaII H \& K lines (Baliunas et al. 1983; Gilliland \& Fisher 1985) can be used, and in eclipsing binaries the rotation rate can be measured by the RossiterMcLaughlin effect or by ellipsoidal light variations. The rotation rate is a well-known quantity for tens of thousands of stars.

DR can only directly be measured on the Sun since moving surface features cannot be resolved on other stars. Fig. 1.1 shows a SOHO/MDI image of the solar surface resolving a large sun spot group and individual active regions. Nonetheless, star spots located at different latitudes are useful tracers for DR (see e.g. Berdyugina (2005); Strassmeier (2009)). Doppler Imaging tracks individual active regions and follows their migration over time to draw conclusions about the stellar rotation law. This method has successfully been used, e.g. by Donati \& Collier Cameron (1997); Collier Cameron et al. (2002). A different technique to measure DR is the Fourier transform method (Reiners \& Schmitt 2002) analyzing the shapes of Doppler broadened line profiles. Analytical spot model (e.g. Budding (1977); Dorren (1987)) have been fit to real data, see e.g. Croll et al. (2006); Fröhlich et al. (2009), accounting for DR in the parameter space. Recently, this method has been used for single Kepler light curves (Frasca et al.|2011; Fröhlich et al. 2012) where DR is the favorite explanation for the light curve shape. Kipping (2012) presents an updated version of the spot model from Budding (1977); Dorren (1987) accounting for DR and spot evolution operating much faster than previous models. Walkowicz et al. (2012) fit an analytical spot model to synthetic light curves of spotted stars to see whether the model can break degeneracies in the light curve, esp. accounting for the ability of determining the correct rotation periods, both in the presence and absence of DR. Following another approach, Lanza et al. (1993) create light curves of spotted stars and detect different periods by taking the Fourier 


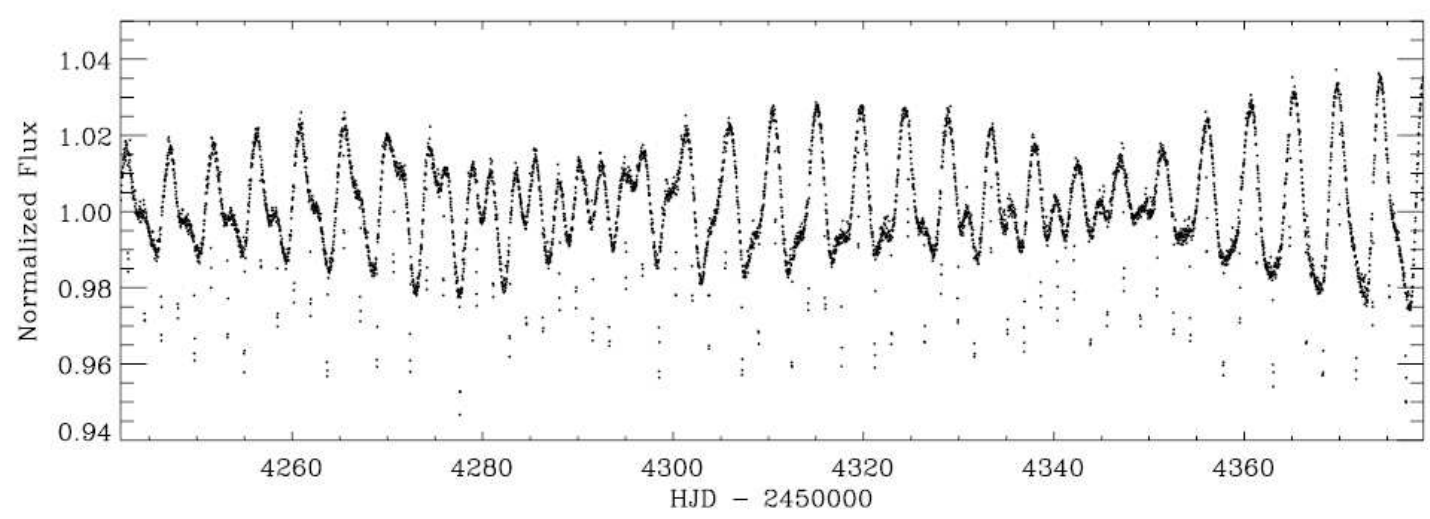

Figure 1.2.: Beat-shaped light curve of CoRoT-2a supplying evidence of differential rotation.

transform. The moderately new field of asteroseismology (Aerts et al. 2010) explains frequency splitting of global oscillations in terms of different latitudinal rotation rates (Gizon \& Solanki) 2004).

\subsection{High precision photometry: CoRoT \& Kepler}

With the advent of the space missions CoRoT2 2 and Kepler photometric data of a vast number of stars has been opened to the public. Both telescopes were originally designed to detect (Earthsized) planets in the habitable zone using the transit method. Thus, they hold an unprecedented precision able to detect the smallest exoplanets, and continuously monitor a wide field of view to increase the probability to detect transiting exoplanets.

Our main focus lies on the detection of rotation periods from co-rotating spots on the stellar surface using them as tracers for surface DR. The detection of DR from photometric data is challenging: To reliably detect a periodic signal in a light curve a good time coverage much longer than the stellar rotation period and a high accuracy are needed - the Kepler telescope provides both of them. The high precision (compared to ground-based observations) is needed to track the behavior of small active regions to study stellar variability at the level of several milli magnitudes. Most of the targets have continuously been monitored for more than 4 years by now. Thus, Kepler not only provides the highest photometric accuracy so far but also the longest photometric data set with the highest duty cycle of more than 160.000 stars.

Fig. 1.2 shows the light curve of the planet host CoRoT-2a. The periodic variability of the light curve is caused by active surface regions rotating in and out of view, and the resulting beating pattern is commonly interpreted as surface DR (Fröhlich et al. 2009; Huber et al. 2010). The CoRoT-2a light curve is an ideal case for detecting DR combining high precision and long-term monitoring. Similar light curve shapes are found quite frequently in Kepler data which was one of the main motivations for this work.

\subsection{Goals and Challenges}

In this thesis I address the problem of measuring surface DR from photometric data of thousands of Kepler stars. To deal with the huge amount of data all light curves are analyzed in an automated way using different algorithms. In this way we try to extract rotation-induced variability from the whole sample. However, in some cases instrumental artifacts due to improper data reduction are mis-interpreted as rotation periods. These cases would have been identified

\footnotetext{
${ }^{2}$ Convection, Rotation, and planetary Transits.
} 
by individual inspection of each light curve which is not possible for tens of thousands of stars. To measure DR from photometric data we developed a fast method that works as follows: We select the active stars from the whole Kepler sample by their amplitude of variability. For these we compute Lomb-Scargle periodograms (Zechmeister \& Kürster 2009) in a prewhitening approach combined with a global sine fit yielding different periods for each light curve. The most significant periods are selected from the fit and associated to surface rotation rates. A similar study has been done by Affer et al. (2012) using Lomb-Scargle periodogram and auto-correlation functions to measure rotation periods for more than 1.700 CoRoT stars. This work is in some sense subsequent to Affer et al. (2012) but focuses on measuring DR facing a much larger data set.

A general problem of light curve analysis is to achieve information about the stellar surface from the one-dimensional time series. Brightness variations are interpreted as different physical processes, like e.g. active regions rotating in and out of view, their evolution over the observing time, pulsations, and instrumental effects. We are only interested in periodic variations caused by co-rotating spots on the surface. Unfortunately, all these effects can introduce (more or less) periodic variations in the light curves.

To distinguish between rotation-induced variability and pulsations is challenging, esp. for $\gamma$ Dor stars (see e.g. Balona et al. (2011a)) showing similar beating patterns as observed for differentially rotating spotted stars (comp. Fig. 1.2). Attempts have been made to classify the whole Kepler sample into known classes of variability like defined pulsation classes (e.g. RR Lyrae, $\delta$ Scuti, etc.), rotation induced variability, binarity, and other groups (Debosscher et al. 2011; Uytterhoeven et al. 2011). In many cases, however, a unique classification is not possible. Furthermore, Kepler only provides broadband light curves in the V-band without further color information. The basic stellar characteristics like $T_{\text {eff }}, \log g$, radius, etc. have been estimated from color information and exhibit large uncertainties, esp. the gravities. For this reason we decided to test the accuracy of our method for spot induced signals alone before applying it to real data. We follow a similar approach to Lanza et al. (1993) running a large Monte-Carlo simulation.

In the following part we explain the model we used to simulate differentially rotating stars (chap. 21), and the method we developed to detect DR (chap. 31). The accuracy of our method is discussed in chap. 4 before applying it to Kepler data (part III). 


Part II.

\section{Simulations}





\section{Simulations}

This chapter explains the model we used to simulate light curves of differentially rotating stars comprising very different properties. First, the model stellar surface is explained. After that I focus on the parameter space for the Monte-Carlo simulation.

\subsection{The Model}

Stellar rotation in the presence of active regions leads to photometric variability in the stars' light curve. MODSTAR is our basic routine that creates a model star to simulate the photometric signal of a rotating spotted star. The star is modeled as a sphere with a fixed resolution of the surface pixels and inclination of the rotation axis. The intensity $I$ and projected area of each surface element depends on the value of $\mu$ which is the cosine of the angle between the pixel's surface normal and the line of sight. A quadratic limb darkening law is used:

$$
I(\mu)=I_{0}\left(1-a(1-\mu)+b(1-\mu)^{2}\right),
$$

with $I_{0}$ being the intensity at the star's center. With respect to the Kepler mission we used the values $a=0.5287$ and $b=0.2175$ from Claret (2000) relating to solar-like stars in the V-band. Active regions can be placed on the model surface. We use circular spots with desired longitudes, latitudes, radii, and a fixed intensity contrast. For the intensity contrast between the spots and the quiet photosphere we use a value of 0.67 which is approximately the solar penumbra to photosphere contrast. For simplicity, all spot pixels have the same contrast value, i.e. we neglect umbra and penumbra structure. The stellar flux is integrated over the whole surface by summing up the pixel intensities weighted by projected area. Since the star can be rotated the flux is calculated at each rotation step which produces a light curve. To describe the implemented rotation law, we quantify the amount of shear by

$$
\alpha=\frac{P_{\text {pole }}-P_{e q}}{P_{\text {pole }}}
$$

with $\alpha=0$ supplying rigid body rotation. $P_{\text {pole }}$ and $P_{e q}$ are the rotation periods at the pole and the equator, respectively. $\alpha>0$ means that the equator rotates faster than the poles (solar-like DR) whereas $\alpha<0$ describes the opposite effect (anti solar-like DR). For our simulations, we only consider solar-like DR since one cannot discriminate between both effects exclusively from the light curve. The rotation period of a spot centered at a certain latitude $\theta$ is given by a common solar-like DR law:

$$
P_{\text {spot }}(\theta)=\frac{P_{e q}}{1-\alpha \sin ^{2}(\theta)}
$$

According to eq.(2.3) a spot would be torn apart after some rotation cycles. To avoid this the spots are fixed on the surface for all phases. In this way we achieve long-lived spots producing a stable beating pattern in the light curve. Evolution of spots is not included in our model so far. The spots are allowed to overlap with no further contrast reduction. 

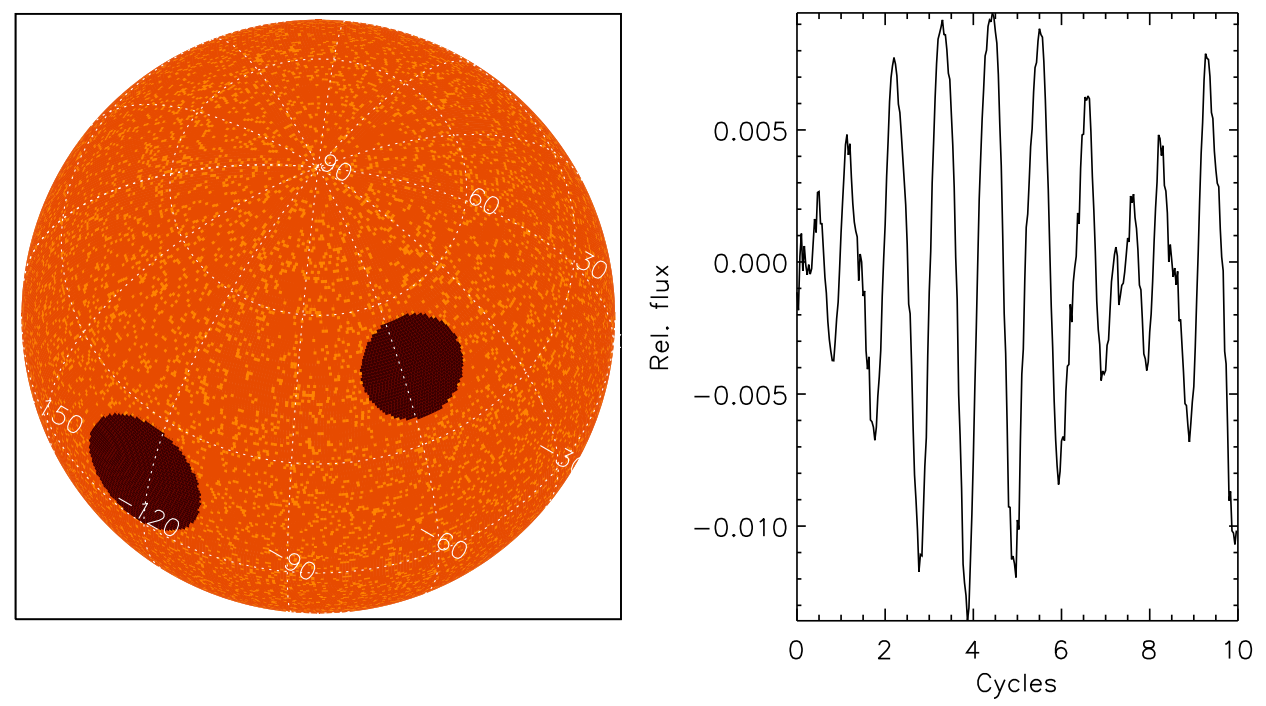

Figure 2.1.: Simulated differentially rotating star with three circular spots and the resulting beat-shaped light curve using MODSTAR.

\subsection{Monte-Carlo simulation}

The Kepler mission provides light curves of all kinds of stellar activity, esp. rotation-induced variability. In some stars DR has been detected (Frasca et al. 2011; Fröhlich et al. 2012), and many other light curves exhibit similar patterns. Inspired by this potpourri of active stars we asked the question to what accuracy DR can be measured solely from photometry if we allow for different kinds of stellar properties and spot configurations.

We ran a Monte-Carlo simulation producing 100.000 light curves of spotted stars to account for a large fraction of possible realizations. The most important stellar parameters are the inclination $i$, the number of spots on the surface, and the amount of DR $\alpha$. All parameters are uniformly distributed with $\sin (i) \in[0,1]$, the number of spots between 1 and 10 , and $\alpha \in[0,1 / 3]$. The inclination covers the whole parameter space from pole-on $\left(i=0^{\circ}\right)$ to edge-on $\left(i=90^{\circ}\right)$ view.

Table 2.1.: Stellar simulation parameters.

\begin{tabular}{lcc}
\hline \hline Parameter & Value & Distribution \\
\hline number of stars & 100.000 & - \\
Inclination $\left[^{\circ}\right]$ & $0-90$ & $\sin (i)$ uniform \\
$\alpha$ value & $0-1 / 3$ & uniform \\
number of spots & $1-10$ & uniform \\
Period $\left[P_{e q}\right]$ & $1-1.5$ & eq.(2.3)
\end{tabular}

The spot positions are chosen at random and the spot radii are between $2^{\circ}$ and $21^{\circ}$ (s. Tables $2.1 \&$ 2.2). The number of spots is limited to 10 because the spot radii can be rather large. These two limits prevent the star from being completely covered with spots which would result in a darker and therefore cooler star. The smaller the spot radii the more pixels are needed in our model to resolve individual surface elements. To limit the computational effort we fixed the minimum spot radius to $2^{\circ}$ since smaller spots require a finer surface resolution.

$\alpha$ covers the parameter space from rigid body rotation $(\alpha=0)$ up to $\alpha=1 / 3$ including the solar 
Table 2.2.: Spot simulation parameters.

\begin{tabular}{lcc}
\hline \hline Parameter & Value $\left.{ }^{\circ}\right]$ & Distribution \\
\hline longitude & $-180-180$ & uniform \\
latitude & $-90-90$ & uniform \\
radius & $2-21$ & uniform
\end{tabular}

value of $\alpha_{\odot}=0.20$. According to eq.(2.3) the period is a function of latitude with $P=1$ cycle at the equator to $P=1.5$ cycles at the poles. A larger $\alpha$ value, e.g. $\alpha=0.5$ would result in rotation periods of $P=1$ cycle at the equator to $P=2$ cycles at the poles. This would lead to problems in discriminating between harmonics and true rotation rates. In observations values of $\alpha>0.50$ have been found (Ammler-von Eiff \& Reiners 2012). Doppler Imaging usually yields lower values of the order $\alpha \lesssim 0.01$, see Table 1 in Barnes et al. (2005), and references therein. Our parameter selection is sufficient to produce light curves of differentially rotating stars for a wide range of $\alpha$. In chap. 4 we will see that the period detection becomes problematic for either very high or very low $\alpha$ values.

The spot to photosphere contrast was fixed because there is a degeneracy between spot size and contrast. Choosing the contrast as free parameter in the simulation would just change the depth of the spot signature in the light curve but does not affect the period. The limb darkening coefficients are fixed for all light curves. Each light curve consists of 300 data points covering 10 rotation periods $P_{e q}$ to see how the light curves evolve in time.

In the following we consider different noise levels in the light curves: the noise-free case, 100 ppm, $1000 \mathrm{ppm}$, and $10.000 \mathrm{ppm}$ Poisson noise which is added to the light curves. A minimum noise level of $100 \mathrm{ppm}$ is chosen because it is lower than the depth produced by the smallest spot $\left(2^{\circ}\right)$ which we find to be approx. $250 \mathrm{ppm}$. The same argument applies to the largest noise level which is lower than the depth of the largest spot $\left(21^{\circ}\right)$ being approx. $48.000 \mathrm{ppm}$.

We want to keep the model simple and try to see whether this can reproduce real light curves. An example of a 1000 ppm model light curve is shown in Fig. 3.1. This light curve looks similar to what we see in Kepler data so we are optimistic that the parameter selection is sufficient for our main purposes - the production of a variety of light curves with periodic variability and the detection of DR. A larger parameter space can be tackled if we find that our model cannot reproduce the data. A similar argument applies to spot evolution, which so far we separate from our approach. 


\section{Period determination}

After the Monte-Carlo simulation we are left with 100.000 light curves. Facing this large sample a fast and reliable frequency analysis tool is needed able to detect DR from the light curve. We chose the Lomb-Scargle periodogram which is widely used in time series analysis since calculating the periodogram of a single light curve takes only one second. Although being a purely mathematical tool the program is sufficient to find different periods in the data. Fitting an analytical spot model also supplies rotation periods and several other stellar parameters but severely slows down the analysis process. In the following we explain our method to extract the most significant periods from the data. This method is first applied to simulated data to test its accuracy before applying it to Kepler data in part III.

\subsection{Lomb-Scargle periodogram}

The Generalised Lomb-Scargle periodogram (Zechmeister \& Kürster 2009) $)^{3}$ is a powerful spectral analysis tool for unevenly sampled data. It fits the data using a series of sines and cosines. The frequency grid used for the fit is sampled equidistantly. Its range has an upper limit due to the Nyquist frequency. The lower limit is given by the inverse product of the time span and a desired oversampling factor to achieve a proper frequency resolution. We use a factor of 10, i.e. a minimum frequency of 0.01 /cycle. Depending on the goodness of the fit one obtains peaks with different powers - the better the fit, the higher the peak in the periodogram. The periodogram is normalized to unity. The period $\mathrm{P}$ (or frequency $\mathrm{f}=1 / \mathrm{P}$ ) associated to the highest peak is the most dominant one in the data. In some cases an alias of $\mathrm{P} / 2, \mathrm{P} / 3$, etc. may produce a peak with high power, too. One reason for a higher alias than the rotational period can be the presence of two active longitudes separated approx. $180^{\circ}$ from each other. Another one can be the improper shape of a sine wave to fit the spot signature. A single spot does not produce a sinusoidal shape except for pole-on view. In frequency domain aliases are equidistant ( $2 \mathrm{f}, 3 \mathrm{f}$, etc.) and can easily be detected by eye because the peak height usually decreases towards higher harmonics. We get rid of most alias periods as described in sec. 3.3 .

\subsection{Prewhitening}

In most cases our model stars are covered by several spots adding up their signals to one light curve. Thus, we are facing the challenge to fit a mixture of periodic signatures. These periods are extracted from the light curve in a successive way called prewhitening. First, we adopt the period associated to the highest peak in the periodogram and fit a sine wave to the light curve. The initial sine function is subtracted from the data and another periodogram is taken from the residuals. Again, we fit a sine function and subtract it from the data. This prewhitening process can be repeated as often as desired, i.e. until there is no periodicity present anymore. On the one hand, a high number of prewhitening steps is crucial for the detection of several periods, but on the other hand, prewhitening is computationally intensive and one has to be careful to select the correct periods afterwards (sec. 3.3) which becomes more difficult with a larger set of periods. Since the stars are covered by 10 spots or fewer we repeat this procedure

\footnotetext{
${ }^{3}$ For different periodogram codes see http://www. astro.physik.uni-goettingen.de/ zechmeister/gls.php
} 


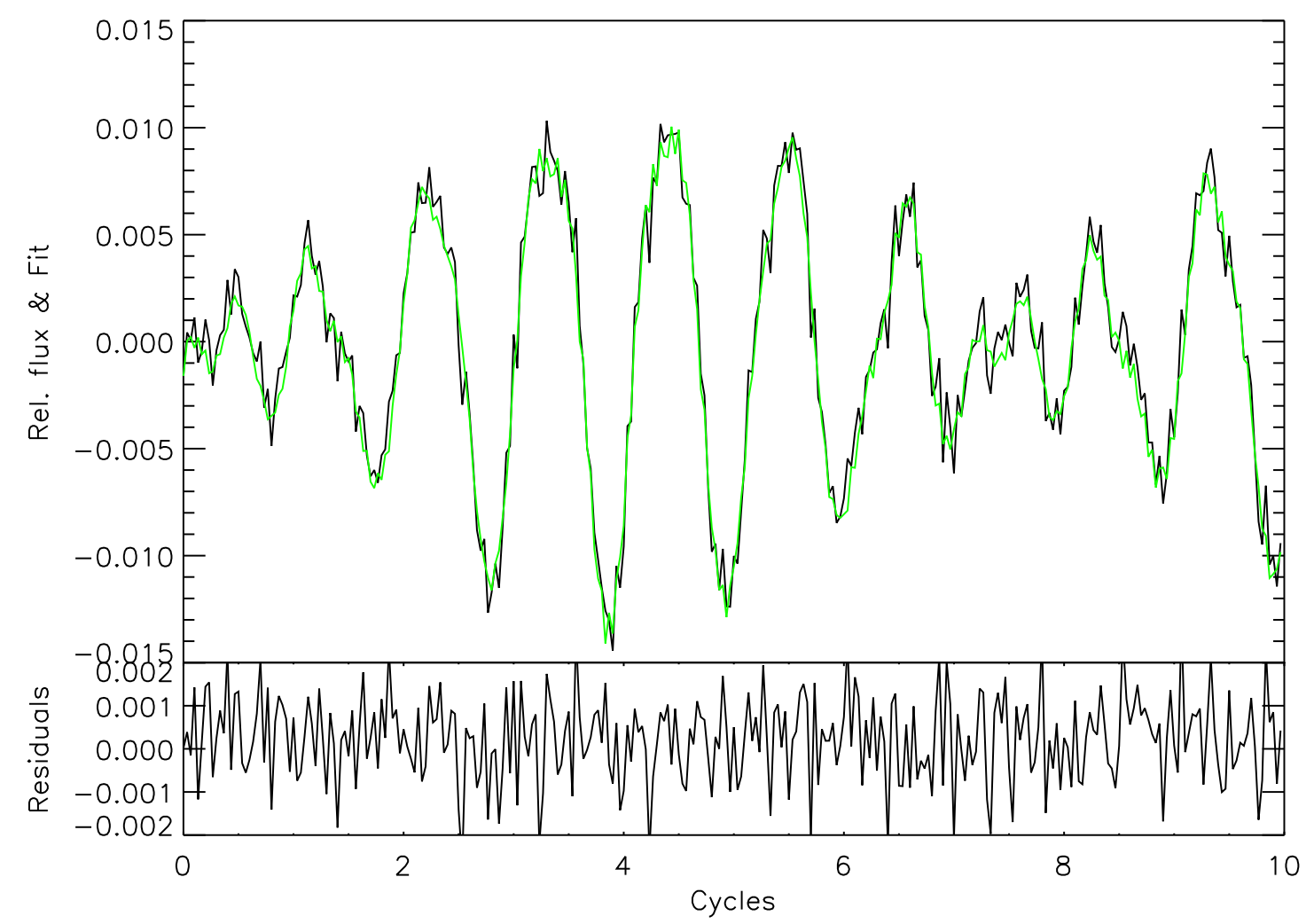

Figure 3.1.: Upper panel: Model light curve from Fig. 2.1 with 1000 ppm Poisson noise added and the best global sine fit over-plotted (green). The spot periods of the model and the ones returned by our method are given in Table 3.1. Lower panel: Residuals of the best fit subtracted from the data; no periodicity visible anymore.

10 times for each light curve. Finally, all 10 periods detected during prewhitening are used as input parameters for a global sine fit, which is the sum of 10 sine functions with different periods, amplitudes, phases, and one total offset. The result of this last step is an optimal set of parameters found through $\chi^{2}$ minimization.

A model light curve with $1000 \mathrm{ppm}$ noise and the fit obtained through this procedure are displayed in Fig. 3.1. In the first column of Table 3.1 the actually contained spot periods are shown; this particular example has only 3 spots. The second column contains all detected periods. The latter ones are arranged as follows: The first period is the one with the highest power found in the prewhitening process, the second one belongs to the second highest power, and so on. We see that in this case the third and fifth period are harmonics of the first and second one, resp., the fourth one and the last five periods just fit the remaining noise. The fit in Fig. 3.1 shows good agreement with the light curve and the residuals carry no more periodicity in the domain of one or more cycles.

\subsection{Period selection}

The fitting procedure described above returns 10 periods for each light curve. In this section we assign a physical meaning to the most significant period (comp. Table 3.1), and show how to detect further periods close to it as evidence for DR. Even though we know that in our model the spot periods range from 1-1.5 cycles (eq 2.3), we will not constrain our algorithm to this 
range since in reality we don't know the correct period either.

The first sine period is the most significant one in the data. In several cases this period is equal to the first harmonic $(\mathrm{P} / 2)$ of the true spot rotation rate due to certain spot configurations, e.g. two spots located on opposite sides of the star. In these cases the second sine period is likely the correct period P. To minimize the number of alias periods we compare the double of the first period to the second sine period. If these two differ by less than $5 \%$ then the second sine period is chosen. The finally selected period is our primary period $P 1_{\text {out }}$. In sec. 4.1 we show that this period yields the best estimate of the stellar rotation rate. In most cases the light curves result from several spots on the surface so we need to compare $P 1_{\text {out }}$ to the spot periods according to eq.(2.3). This set is called the input periods since they belong to spots inherent in the light curves. Thereof, we take the one closest to $P 1_{\text {out }}$ and call it $P 1_{\text {in }}$.

Based on the period $P 1_{\text {out }}$ we looked for a second period which we call $P 2$ for the moment. In order to attribute this period to a second spot one has to balance three things: 1) Find a second period that is no harmonic of $\left.P 1_{\text {out }}, 2\right)$ try to exclude as few spot periods as possible, and 3 ) try to dismiss all period artifacts that come from fitting a sine wave to the light curve rather than a spot model. Therefore, $P 2$ should hold the relations

$$
0.01 \leq \frac{\left|P 1_{\text {out }}-P 2\right|}{P 1_{\text {out }}} \leq \alpha_{\text {max }}
$$

with $\alpha_{\max }=1 / 3$. The value $\alpha_{\max }=1 / 3$ corresponds to the maximum $\alpha$ in our model. As mentioned above a higher value near $\alpha_{\max } \approx 0.5$ would yield ambiguous results for the DR. Image two cases: 1) $P 1_{\text {out }}=1, P 2=0.5$, and 2) the case $P 1_{\text {out }}=1, P 2=1.5$. In both cases a second period would be selected but due to completely different origins. In case 1) the first harmonic of $P 1_{\text {out }}=1$ would be mis-interpreted as DR, whereas case 2) results from real spot configurations. We chose the value $\alpha_{\max }=1 / 3$ because it excludes harmonics and covers a wide range for a second spot period. For example, in the extreme case of $P 1_{\text {out }}=1$, the harmonic at $P 2=1 / 2$ is excluded but we are not able to find a spot period greater than $P 2=1.33$ although there might be spots with longer periods. The lower limit in relation (3.1) accounts for the fixed frequency resolution in the Lomb-Scargle periodograms (s. sec. 3.1). If two spot periods differ by less than $1 \%$ they cannot be resolved. Again, we might miss some spot periods lying closer than $1 \%$ with our method.

If one or more periods were found fitting both criteria (compare Table 3.1) we took that P2 associated to the period with the lowest row index in the table of remaining sine periods. This period is called $P 2_{\text {out }}$. In 88330 cases of our models we find a $P 2_{\text {out }}$ that fulfills these criteria. Again, $P 2_{\text {out }}$ is compared to all input periods and the period closest to $P 2_{\text {out }}$ is called $P 2_{\text {in }}$. If the closest input period picked is again $P 1_{\text {in }}$ then $P 2_{\text {out }}$ is discarded. Finally, we are left with 64172 stars having two periods which belong to two different spots.

For our example light curve in Fig. [3.1, the periods $P 1_{\text {out }}=1.016, P 2_{\text {out }}=1.184, P 1_{\text {in }}=1.018$, and $P 2_{\text {in }}=1.186$ have been selected from Table 3.1. Calculating the relative shear of the two spots (comp. eq. 4.1) results in $\alpha_{\text {in }}=0.14$ and $\alpha_{\text {out }}=0.14$. Although our method is able to recover the correct $\alpha_{i n}$ value, the total equator-to-pole shear equals $\alpha=0.30$ in this case (comp. eq.(2.3) $)$, and thus is underestimated by more than $50 \%$. For this specific spot configuration it is impossible to obtain the correct $\alpha$ value since the highest spot latitude equals $\theta=46.3^{\circ}$ generating the longest period. This is a general problem of DR measurements from photometric observations due to the initial spot configuration on the surface. The measured shear will always yield a lower value than the total one.

\footnotetext{
${ }^{4}$ If $P 1_{\text {out }}$ was a harmonic then the first two sine periods are excluded.
} 
Table 3.1.: Period selection process using the example of the light curve from Fig. 3.1, Left: Periods of the three spots from the light curve in Fig. 3.1 (left column) and output periods returned by the prewhitening analysis for the fit in Fig. 3.1 (right column). Upper right: The two periods $P_{\text {in }}$ and $P_{\text {out }}$ that have been selected from the left table. Lower right: The resulting values $\alpha_{\text {in }}$ and $\alpha_{\text {out }}$ computed from the upper table (comp. eq. 4.1).

\begin{tabular}{|c|c|c|c|c|}
\hline$P_{\text {spot }}$ & $P_{\text {out }}$ & & $P_{i n}$ & $P_{\text {out }}$ \\
\hline 1.049 & 1.016 & & $\frac{1 n}{1.018}$ & 1.016 \\
\hline 1.018 & 1.184 & $\Longrightarrow$ & 1.186 & 1.184 \\
\hline 1.186 & 0.510 & & & \\
\hline - & 0.906 & & & \\
\hline - & 0.591 & & & \\
\hline- & 0.078 & & & \\
\hline- & 0.081 & & $\alpha_{i n}$ & $\alpha_{\text {out }}$ \\
\hline- & 0.340 & & 0.14 & 0.14 \\
\hline- & 0.079 & & & \\
\hline - & 0.134 & & & \\
\hline
\end{tabular}

\subsection{Sample Properties}

This section is thought as a consistency check of our model and the selection algorithm. The stellar parameters (s. Table 2.1) of two mutually exclusive samples, S2 and S1, are compared. The S2 sample consists of all light curves with two detected periods (64.2\%) coming from two distinct spots, whereas S1 contains all cases where only one spot period could be associated $(32.0 \%)$. Due to a combination of a low number of spots, certain spot latitudes, and a low inclination in $3.8 \%$ of all cases no spot was visible.

The goal is to point out those stellar models where DR can likely be found compared to the cases where the detection of DR is challenging or even impossible with our method. For example, one would expect that it is easier to detect DR in the case of a highly spotted star rather than in the case of a star covered by only two close-in spots because it will probably be a heavy task to resolve individual periods in the latter case.

In Fig. 3.2 we compare the inclination, number of spots, spot radii, and differential rotation $\alpha$ of both samples. The S2 sample is shown in the left and the S1 sample in the right column, resp.. The colors correspond to the different noise levels: noise-free (green), $100 \mathrm{ppm}$ (yellow), 1000 ppm (orange), and $10.000 \mathrm{ppm}$ (red) Poisson noise. Due to the small difference of detections for the noise-free (green) and the $100 \mathrm{ppm}$ noise case (yellow) there is basically no difference visible in the histograms. All trends have similar shape and become more distinct towards higher noise levels.

In the first row the inclination for both samples is plotted. Remember that $\sin (i)$ of the whole sample has a flat distribution. Starting from edge-on view $\left(i=90^{\circ}\right)$ we see a continuous decrease (increase) of detections in the S2 (S1) sample. Around inclinations lower than $i=10^{\circ}$ the number of detections in S2 decreases significantly. The opposite effect applies to S1.

In the second row we show distributions of the actual number of spots of the models. We find that only in a very few cases the models in S2 can be attributed to only two spots on the surface. In the majority of all cases the light curve is composed by the signature from more than 5 spots! We find that the actual number of spots decreases in the S1 sample.

The third row shows the distribution of the spot radii. In the left panel one clearly sees that the radii of both spots found increases to higher values because it is more likely to detect more 

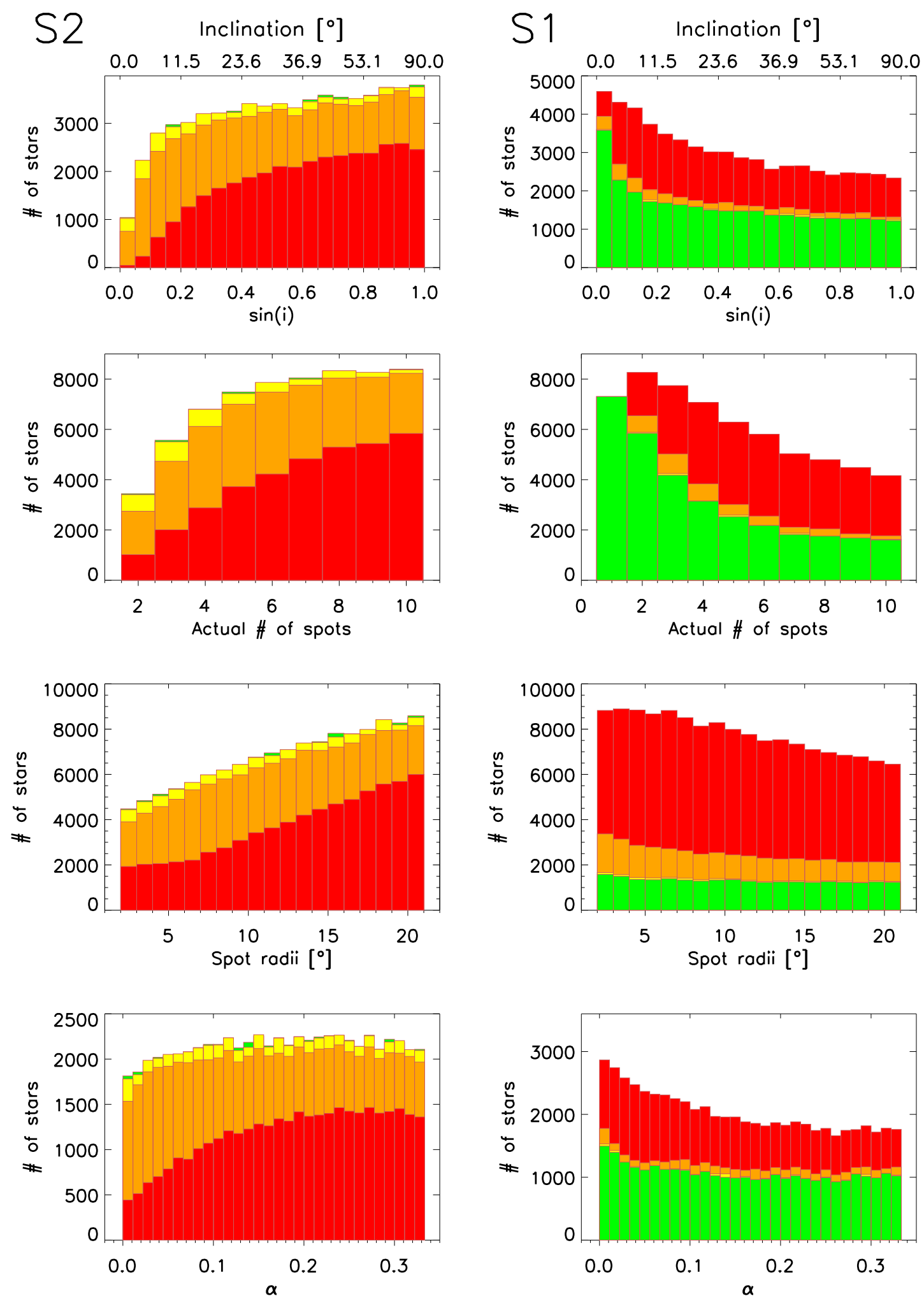

Figure 3.2.: Comparison of basic stellar parameters for both samples S2 and S1 for different noise cases: noise-free (green), 100 ppm (yellow), 1000 ppm (orange), and 10.000 ppm (red) Poisson noise. Left panel: Stars with two spot periods (S2). Right panel: Stars with only one spot period (S1). 
than one period if the star has large spots. In the right panel we plot the radii of all visible spots except for the one associated to the one period found. These "residual" or not resolved spots show a shallow decrease in radii.

Finally, the last row shows histograms of the $\alpha$ value. Both distributions are basically flat except for the $10.000 \mathrm{ppm}$ noise case and for small values of $\alpha$. The distribution of S2 decreases while the one of $\mathrm{S} 1$ increases towards lower $\alpha$ values.

All above histograms show consistent results for each sample supporting the underlying model. With a focus on the S2 sample the selection process seems convincing to pick mostly those models where the detectability of DR is expected. The derived periods and accuracy of our method are discussed in the following section. 


\section{Simulation Results}

In this chapter we compare the outcome of our method to the periods from our model. First, we present the basic results for the most dominant period $P 1_{\text {out }}$. In sec. 4.2 the detection of DR is discussed considering different noise levels.

\subsection{Rotation periods $P 1_{\text {out }}$}

In $96.2 \%$ of all light curves there is one detected periodicity $P 1_{\text {out }}$ which is the most significant one in the data. In Fig. 4.1 we compare the periods $P 1_{\text {out }}$ to the input periods $P 1_{\text {in }}$ for different noise levels to see how good the above selection process works. The distribution of input periods $P 1_{\text {in }}$ is shown in gray, and the shaded distribution shows the output periods $P 1_{\text {out }}$. Since we only consider solar-like DR $P 1_{\text {in }}$ cannot be lower than 1 cycle. The shaded gray area shows that both histograms overlap quite well. For the noise-free case the number of wrong detections is negligible with $3.3 \%$ of all periods being lower 0.9 cycles and $2.9 \%$ being greater than 1.6 cycles. This is no longer true for higher noise levels. The region from $0-0.5$ cycles becomes populated as shown in the 3 small plots in Fig. 4.1,

We compare the weighted means $\left\langle P 1_{\text {in }}\right\rangle$ and $\left\langle P 1_{\text {out }}\right\rangle$ in the above range in Table 4.1. In the noise-free case we find that $P 1_{\text {out }}$ is on average $2.4 \%$ lower than the actually contained period $P 1_{\text {in }}$. For higher noise levels $\left\langle P 1_{\text {out }}\right\rangle$ decreases because the periodogram interprets the noise as short periods. In the noise-free case the wrong detections are due to a low stellar inclination close to pole-on view or in some cases due to higher harmonics. The cases where $P 1_{\text {out }}$ is lower than 1 cycle are due to the improper shape of a sine function to fit spot signatures in a light curve. For example, a detected period of $P 1_{\text {out }}=0.98$ cycles will be considered as valid rotation period although it is not possible for the spots to rotate this fast in our model. This fact will not be noticed in real data because we do not have information on the real rotation of a star. Around a period of 0.5 cycles only a small fraction of harmonics remained (less than $0.5 \%$ ) after identification and correction (s. sec. 3.3).

\subsection{Differential Rotation}

In this section we show in which situations DR can successfully be detected and which cases lead to wrong interpretations. In our model the detection of a second period $P 2_{\text {out }}$ adjacent to

Table 4.1.: Weighted means $\left\langle P 1_{\text {in }}\right\rangle$ and $\left\langle P 1_{\text {out }}\right\rangle$, and their associated errors $\sigma\left(P 1_{\text {in }}\right)$ and $\sigma\left(P 1_{\text {out }}\right)$ for both input and output periods, resp. for each noise level.

\begin{tabular}{ccccc}
\hline \hline Noise $[\mathrm{ppm}]$ & $\left\langle P 1_{\text {in }}\right\rangle$ & $\sigma\left(P 1_{\text {in }}\right)$ & $\left\langle P 1_{\text {out }}\right\rangle$ & $\sigma\left(P 1_{\text {out }}\right)$ \\
\hline 0 & 1.066 & 0.094 & 1.042 & 0.160 \\
100 & 1.066 & 0.094 & 1.039 & 0.168 \\
1000 & 1.066 & 0.094 & 1.018 & 0.216 \\
10.000 & 1.058 & 0.089 & 0.843 & 0.394
\end{tabular}




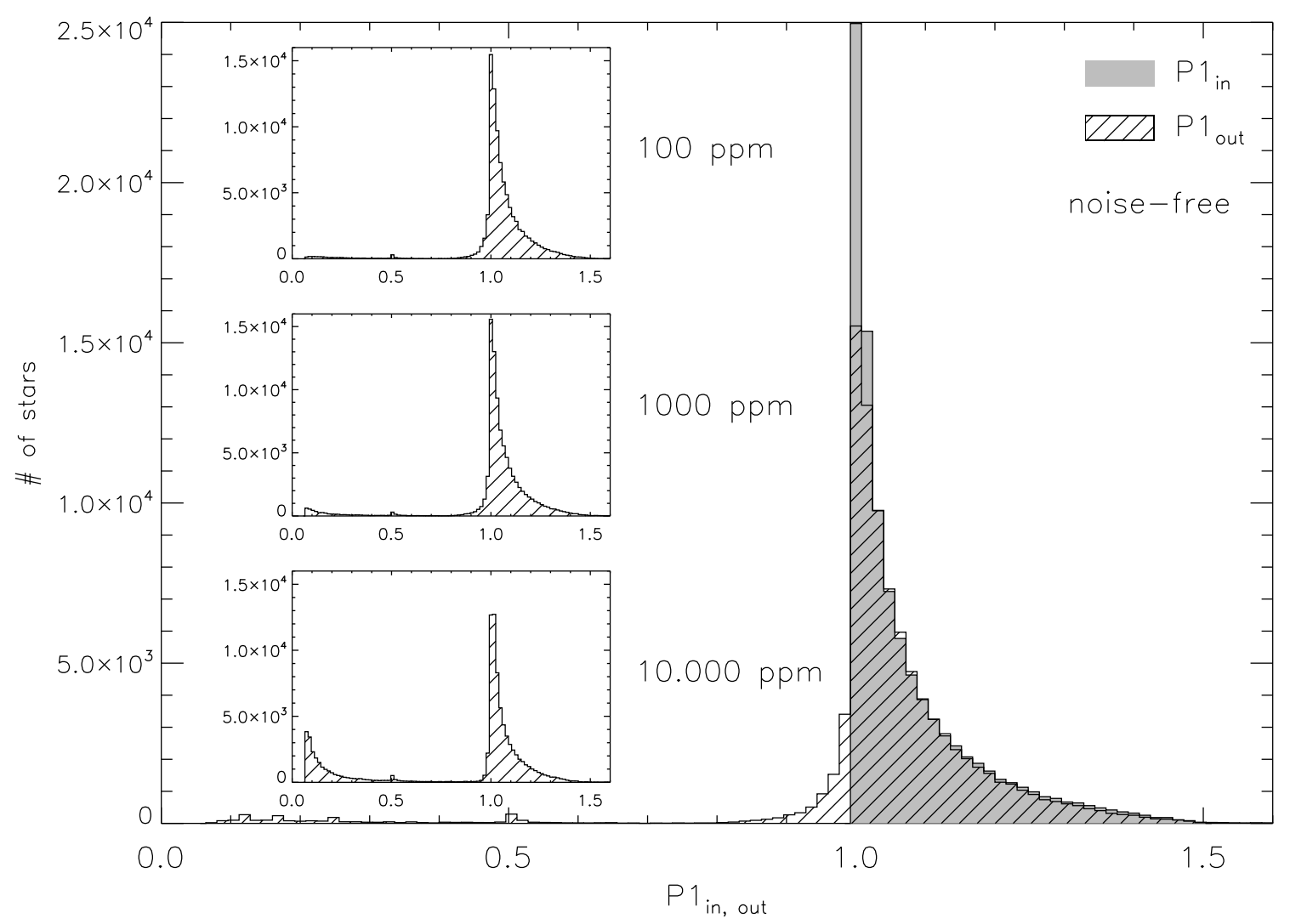

Figure 4.1.: Main plot: Comparison of exactly known input period $P 1_{\text {in }}$ and output period $P 1_{\text {out }}$ for the noise-free case. We see that the most significant period $P 1_{\text {out }}$ can be recovered fairly well. We find that $P 1_{\text {out }}$ is on average $2.4 \%$ lower than the actually contained period $P 1_{\text {in }}$. Small plots: The distribution of $P 1_{\text {out }}$ is shown with increasing noise from top to bottom. Towards higher noise levels the fraction of periods lower than 0.5 cycles increases because the algorithm interprets the noise as short periods (comp. Table 4.1).

the primary one and associated to a second spot period $P 2_{i n}$ is considered as evidence for DR. To see where this selection process is acceptable we consider the two pairs $\left(P 1_{\text {out }}, P 2_{\text {out }}\right)$ and their associated periods $\left(P 1_{i n}, P 2_{i n}\right)$. We estimate the amount of DR by sorting these pairs and computing their $\alpha_{\text {in, out }}$ value, resp. (s. Table 3.1):

$$
\alpha_{\text {in }, \text { out }}=\left.\frac{P 1-P 2}{P 1}\right|_{\text {in,out }}, P 1>P 2 .
$$

The $\alpha_{i n}$ value is always lower than the inherent $\alpha$ value of each light curve since we can only measure the rotational shear at two defined latitudes. If $\alpha_{i n}$ is calculated from the spots with the largest separation in latitude on a certain hemisphere then $\alpha_{i n}$ is the maximum shear that can be detected by our method. The distribution of $\alpha_{\text {out }}-\alpha_{\text {in }}$ gives a statistical measure of the robustness of our period selection process. In Fig. 4.2 we show the distribution of the differences between $\alpha_{\text {out }}$ and $\alpha_{\text {in }}$ in our set of models for different noise levels. All distributions exhibit an asymmetric shape towards too large $\alpha_{\text {out }}$ values. An explanation for $\alpha_{\text {out }}$ being too large is given at the end of this section. The total number of light curves with two (or more) detected periods, the weighted mean, and the error of the distributions are given in Table 4.2. In general, the total number of stars with two detected periods decreases with increasing noise. All differences between the noise-free and the $100 \mathrm{ppm}$ case are marginal. The $1000 \mathrm{ppm}$ case has slightly less detections, and the most significant decrease happens for the $10.000 \mathrm{ppm}$ case (compare 

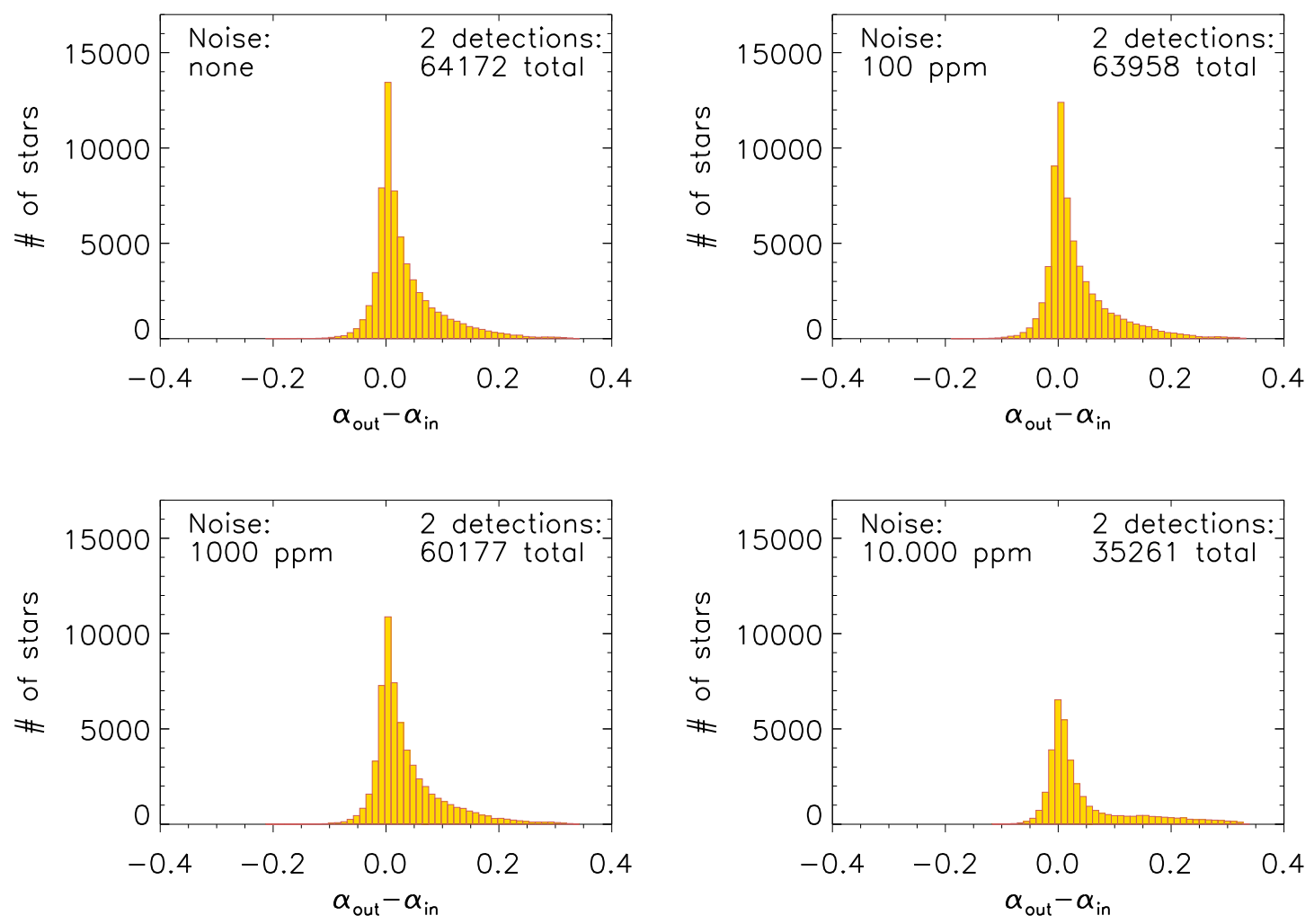

Figure 4.2.: Distribution of $\alpha_{\text {out }}-\alpha_{\text {in }}$ for each noise level. From the upper left to the lower right panel the noise increases. For the noise-free case (upper left panel) in 64172 stars (at least) two periods are detected. The distribution is centered at $\left\langle\alpha_{\text {out }}-\alpha_{\text {in }}\right\rangle=0.032$ with a width of $\sigma\left(\alpha_{\text {out }}-\alpha_{\text {in }}\right)=0.058$. Going to higher noise levels the total number of findings decreases and the error increases (s. Table 4.2).

Fig. 3.2). For all cases the weighted mean and the error increase with noise. Considering the noise-free distribution (upper left) we find a weighted mean $\left\langle\alpha_{\text {out }}-\alpha_{i n}\right\rangle=0.032$ and a width of $\sigma\left(\alpha_{\text {out }}-\alpha_{\text {in }}\right)=0.058$. The error is given in absolute units of $\alpha_{\text {out }}-\alpha_{\text {in }}$ regardless of the true shear $\alpha$. We also considered the relative errors $\left|\alpha_{\text {out }}-\alpha_{\text {in }}\right| / \alpha$ for $\alpha>0.05$. Each distribution is proportional to const. $/ \alpha$, i.e $\sigma\left(\alpha_{\text {out }}-\alpha_{\text {in }}\right)$ does not scale with $\alpha$. For each noise level the number of stars decreases with increasing relative error. We only considered $\alpha>0.05$ to avoid large errors. The statistical error resulting from the sample of the light curves used is negligible. The above calculations have been done for two additional sets of light curves (each 100.000 in total). We find that $\left\langle\alpha_{\text {out }}-\alpha_{\text {in }}\right\rangle$ and $\sigma\left(\alpha_{\text {out }}-\alpha_{\text {in }}\right)$ are almost equal for each set, and that the largest statistical uncertainty in the number of stars with two detected periods is about $0.3 \%$.

We have shown that DR can be measured with high accuracy for a large noise range. In Fig. 4.3 we compare $\alpha_{\text {out }}$ to the total equator-to-pole shear $\alpha$ of the star. In the noise-free case the distribution has a weighted mean $\left\langle\alpha_{\text {out }}-\alpha\right\rangle=-0.056$ and an error $\sigma\left(\alpha_{\text {out }}-\alpha\right)=0.098$. Considering the mean values yields

$$
\begin{aligned}
0.032 & =\left\langle\alpha_{\text {out }}-\alpha_{\text {in }}\right\rangle \geq\left\langle\alpha_{\text {out }}-\alpha\right\rangle=-0.056 \\
& \Rightarrow\left\langle\alpha_{\text {out }}-\alpha\right\rangle \leq 0.088
\end{aligned}
$$

since $\alpha_{i n} \leq \alpha$ by definition. This means that the total amount of DR is underestimated by $8.8 \%$ by our method. Models with small $\alpha$ values exhibit spot periods very close to each other. 


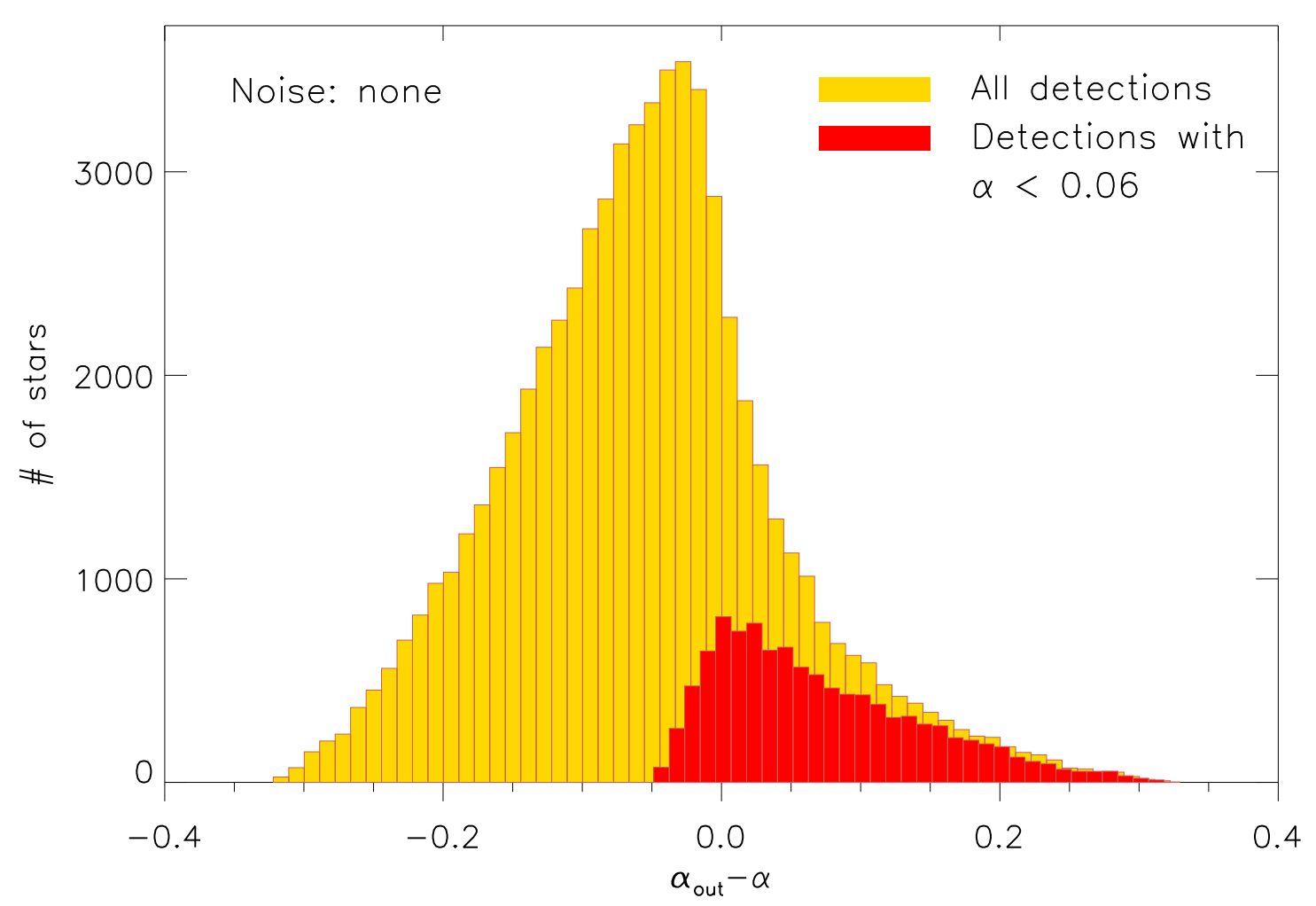

Figure 4.3.: Distribution of $\alpha_{\text {out }}-\alpha$ for the noise-free case centered at $\left\langle\alpha_{\text {out }}-\alpha\right\rangle=-0.056$ with a width of $\sigma\left(\alpha-\alpha_{\text {out }}\right)=0.098$. Comparing this plot to Fig. 4.2 shows that the total amount of $\mathrm{DR}$ is underestimated by $8.8 \%$. Models with $\alpha<0.06$ are over-plotted in red. It is evident that in these cases the detection of DR is difficult. In most cases, $\alpha_{\text {out }}>\alpha$ demonstrating the limits of our method.

Thus, we tested whether these are prone to mis-identification of DR. In Fig. 4.3 the models with an equator-to-pole shear $\alpha<0.06$ are over-plotted in red. We find that $\alpha_{\text {out }}$ yields wrong values larger than $\alpha$ itself. This results from the difficulty to resolve two distinct peaks in the Lomb-Scargle periodogram. Since the peak width is proportional to the inverse time span of the light curve we are not able to resolve two periods within 10 cycles. One broadened peak appears resulting in a mean period. In these cases the initial sine wave does not have a proper shape to fit the mixture of spot signatures so there remain artifacts which are corrected by fitting more sine waves. If one of the residual periods fulfills the selection criteria the algorithm selects it as $P 2_{\text {out }}$ yielding too high $\alpha_{\text {out }}$ values. This behavior partly applies to the distributions in Fig. 4.2, esp. for the highest noise case. A second spot period cannot be resolved properly and is lost in the noise yielding an asymmetric distribution.

We have seen that our method is able to detect DR with relatively high accuracy in simulated data. Thus, we apply our method to the active Kepler stars in the following part. 
Table 4.2.: Weighted mean $\left\langle\alpha_{\text {out }}-\alpha_{\text {in }}\right\rangle$ and error $\sigma\left(\alpha_{\text {out }}-\alpha_{\text {in }}\right)$ of the distributions in Fig. 4.2,

\begin{tabular}{cccc}
\hline \hline Noise $[\mathrm{ppm}]$ & \# of stars & $\left\langle\alpha_{\text {out }}-\alpha_{\text {in }}\right\rangle$ & $\sigma\left(\alpha_{\text {out }}-\alpha_{\text {in }}\right)$ \\
\hline 0 & 64172 & 0.032 & 0.058 \\
100 & 63958 & 0.033 & 0.059 \\
1000 & 60177 & 0.036 & 0.061 \\
10.000 & 35261 & 0.044 & 0.078
\end{tabular}
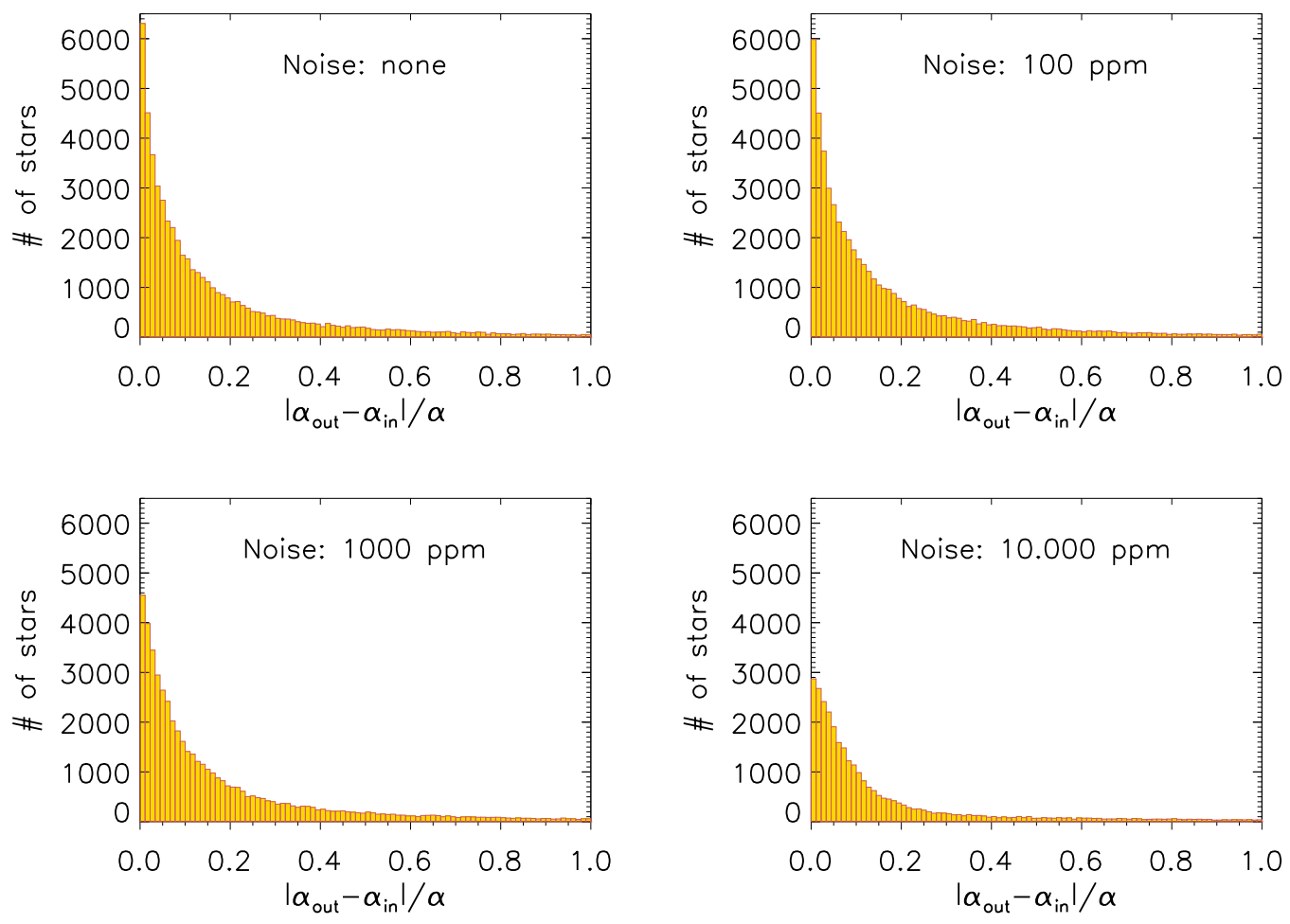

Figure 4.4.: Distribution of the relative error $\left|\alpha_{\text {out }}-\alpha_{\text {in }}\right| / \alpha$ for $\alpha>0.05$. From the upper left to the lower right panel the noise increases. Each distribution is proportional to const./ $\alpha$, i.e. with increasing error the number of stars decreases regardless of $\alpha$. We only consider $\alpha>0.05$ because small $\alpha$ values yield very large errors. 


Part III.

\section{Kepler data}





\section{Selection of active stars}

Stellar activity covers a wide field of different activity phenomena. Our main focus lies on the detection of periodic variability induced by dark spots co-rotating with the star. Since the spot periods constrain the stellar rotation law the detection of more than one dominant period is considered as hint for DR although there might be other effects able to mimic DR. In Fig. 5.1

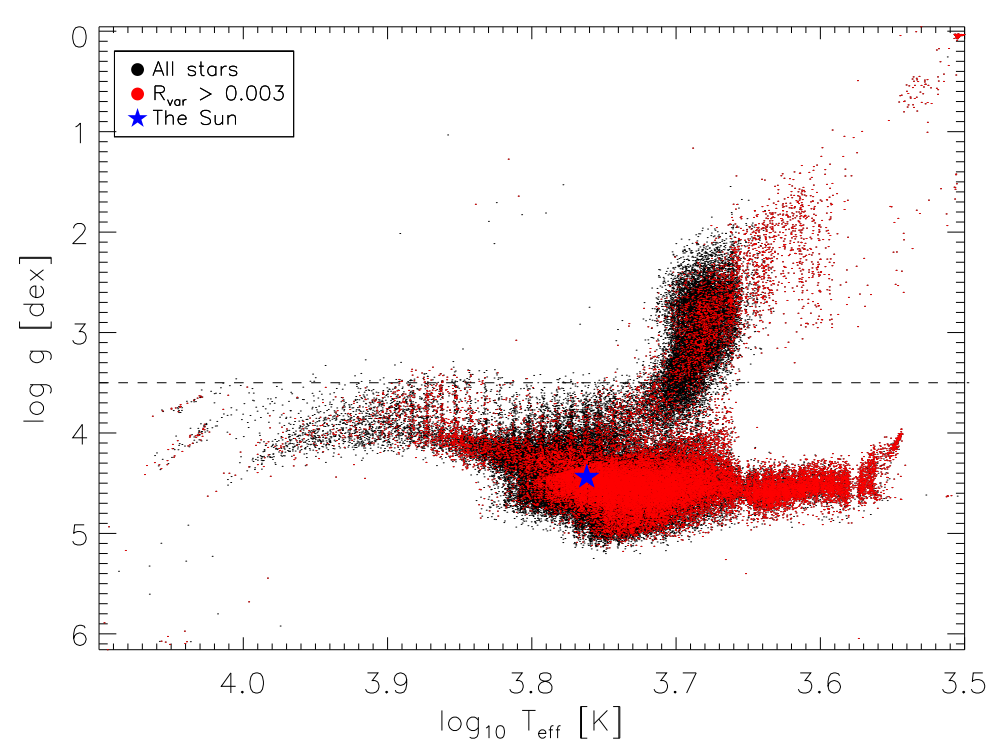

Figure 5.1.: Temperature vs. gravity of all Kepler Q3 stars (black) using KIC parameters. The active sample $\left(R_{v a r} \geq 0.003\right)$ is shown in red. The blue star marks the Sun which is shown for comparison. The dashed line marks $\log g=3.5$ which is set to exclude giants in the following.

we plot effective temperature vs. gravity of the whole Kepler Q3 sample (black dots), with the active stars shown in red. The selection of active stars is done automatically, i.e. without visual inspection of the light curves since the Kepler sample is huge. The active stars are selected using the so-called variability range $R_{\text {var }}$ (Basri et al. 2010, 2011). The value is computed as follows: We sort the 4 hours boxcar smoothed differential light curve by amplitude, cut the upper and lower 5\%, and take the difference between top and bottom amplitude. This measure accounts for the intrinsic variability of the star, i.e. a variable star has a larger variability range than a quiet star. After visual inspection of several light curves we found that a suitable criterion is $R_{v a r} \geq 0.003$ (3 parts per thousand).

Most of the active stars populate the dwarf regime with $\log g \gtrsim 4$. The upper right corner shows a significant fraction of active cool stars with $\log g \lesssim 3$. Visual inspection of these low gravity stars reveals two groups of variability. The first one has very high ranges up to several percent, regular spot-like variations, and long periods. This might indicate spots or pulsations on giants, which we do not consider in this work. The second group exhibits irregular variability on short time scales which could be due to non-radial pulsations. The sun (blue star) is shown for comparison. All parameters have been taken from the Kepler Input Catalog (KIC). They have been estimated from color information and might contain large errors for individual targets. 


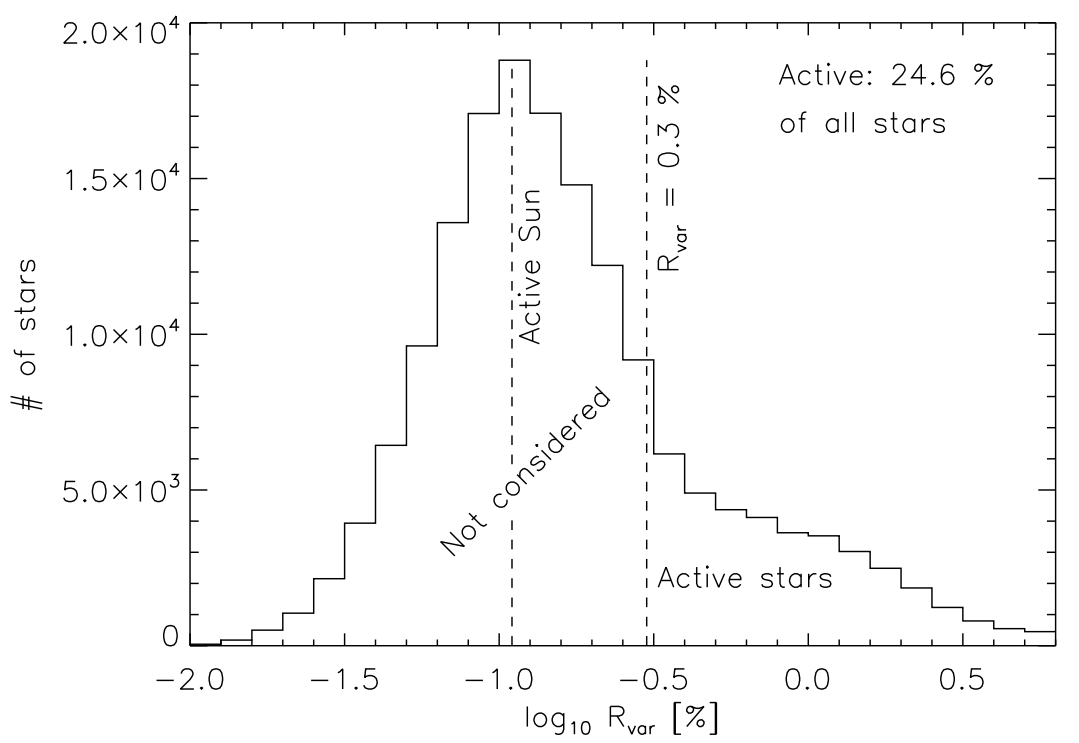

Figure 5.2.: Distribution of $R_{v a r}$ for all Kepler Q3 stars. The lower limit to $R_{v a r}$ excludes more than $75 \%$ of all stars.

We see that the Kepler sample is strongly biased towards solar-like stars, but also a large giant branch $(\log g \lesssim 3.5)$ is clearly visible. In the following we will only consider stars lying under the dashed line hoping to exclude most of the giants.

We calculated the variability range of the sun from to total solar irradiance (TSI) data from the VIRGO instrument at the SOHO satellite, and compared it to our limit $R_{v a r} \geq 0.003$. Using data from 1 Dec 1995 to 1 Sep 2011 we found that the maximum range was $\max \left(R_{v a r, \odot}\right)=0.0023$, with a mean of $\left\langle R_{v a r, \odot}\right\rangle=0.0011$ during solar maximum (Feb 201999 - Oct 21 2004). This value lies below our limit, thus all stars considered are more active than the active sun. The variability range is our key measure to distinguish between active and quiet stars, i.e. all stars with $R_{v a r}$ above the upper limit will be called active although there is a large spread in their ranges. 40.661 stars of the whole Kepler sample survive this criterion. The distribution of ranges is shown in Fig. 5.2. The peak of the range distribution lies at the value of the active sun, in agreement with the majority of the sample being solar-like stars. Only $24.6 \%$ of all stars are considered as active, i.e. to these stars we will apply the analysis procedure from part II. 


\section{Period determination II}

In this chapter we briefly summarize the method from chapter 3 to achieve the most significant period from a given light curve. We slightly changed our method to deal with the huge amount of Kepler data. The changes do not alter the basic idea of the method but only account for computational efficiency. The period selection also slightly differs from the one in sec. 3.3 since we have to account for other types of (periodic) variability.

\subsection{Lomb-Scargle periodogram \& Pre-whitening}

As described in chapter 3 we use the Generalised Lomb-Scargle periodogram (Zechmeister \& Kürster 2009) to detect periods in a light curve. To save computation time each light curve was binned to 2 hour bins. Here, the lowest frequency is given by the inverse product of the time span $(\approx 90$ days) times an oversampling factor of 20 , i.e. $f_{\text {low }} \approx 1 /(90 \cdot 20 d)$, which accounts for proper frequency sampling. Using a denser frequency sampling (factor $=30$ ) does not significantly change the results. The highest frequency is given by the Nyquist frequency using the above binning. The binning will not affect the period determination since we only consider periods longer than half a day (sec. 6.2).

For Kepler data the pre-whitening procedure is repeated only five times rather than ten times. Visual inspection of several light curves confirmed that the resulting fit is sufficient to detect significant rotation-induced periods. Using more pre-whitening steps results in a better fit but is computationally intensive and does not yield significantly different periods. The enlarged set of returned periods makes it more difficult to assign a physical meaning to the individual periods (sec. 6.2). For some very active stars with regular beating pattern in their light curve there might be more than five periods that could be assigned to active regions on the surface. Five steps is a good compromise between goodness of the fit and computational efficiency. Three example light curves, the resulting fits, and the corresponding periodograms are discussed in section 7.3 .

\subsection{Period selection}

The next step is to select the most significant periods from the global sine fit, and to assign a physical meaning to them. We are interested in rotation periods of the star. The whole concept of only one rotation period is not quite exact because one can only detect periodic variations caused by active regions located at certain latitudes. We think of either single spots or spot groups rotating over the visible hemisphere. If these regions are not located at the equator then the equatorial rotation period remains unknown. Another problem arises from stars with a high spot coverage due to many small spots. If they are inhomogeneously distributed over both sides of the star the light curve cannot be distinguished from a star with few active regions. Nevertheless, we use the first sine period from the global sine fit as the most significant period in the light curve. This period belongs to the highest power found in the pre-whitening process, and is therefore defined as one rotation period. In some cases the spots are located on opposite sides of the star, and the half period is what is detected. To minimize these alias periods we compare the first two periods from the global sine fit. If the difference of twice the first period 
and the second period is less than $5 \%$ then the algorithm selects the longer one which is likely the correct period. The period finally selected is our primary period $P_{1}$.

If the star rotates differentially, active regions have different velocities which manifests in a superposition of different periods in the light curve. To search for a second period close to $P_{1}$ we look for a period $P_{2}$ within $30 \%$ of $P_{1}$ in the remaining 4 sine periods. To estimate the relative surface shear of the two active regions we define

$$
\alpha:=\left|P_{2}-P_{1}\right| / P_{\max }
$$

with $P_{\max }=\max \left\{P_{1}, P_{2}\right\}$. Since we cannot tell from the light curve whether the star rotates solar-like or anti solar-like we always assume solar-like DR. To get closest to the total equatorto-pole shear we normalize the period difference by $P_{\max }$. Equivalent to $P_{\max }$ we define $P_{\min }=$ $\min \left\{P_{1}, P_{2}\right\}$ to get closest to the equatorial period. $\alpha$ should hold the relations

$$
\alpha_{\min } \leq \alpha \leq \alpha_{\max }
$$

The upper limit $\alpha_{\max }=0.3$ is a reasonable choice since the solar value is $\alpha_{\odot}=0.2$ and the total amount of DR is unknown for stars other than the sun. In sec. 8.6 we show that the general results are not affected by using different $\alpha_{\max }$ values. The lower limit $\alpha_{\min }=0.01$ accounts for the fixed frequency resolution of the periodograms. If there are more than one sine periods satisfying both criteria then the one with the second highest power in the pre-whitening process is chosen. If $P_{1}$ was an alias period then only the remaining 3 sine periods are considered to look for a second period.

In contrast to previous studies we find that the highest peak of the initial periodogram is a bad measure to filter out active stars. The periodogram often detects a period longer than 90 days. These long periods remain doubtful because one cannot distinguish between remnants from the PDC-MAP pipeline and real long-term variability. Their peak height in the periodogram is similar to more reliable shorter periods, i.e. the peak height is highly biased by the data reduction. Thus, the variability range is the only measure we use.

Now, we make further restrictions to the derived periods to assure that these are really due to rotation. Using the variability range we selected 40.661 active stars from the whole sample. As seen in Fig. 5.1 there are several active giants. Since we are mainly interested in rotation periods of main-sequence stars the surface gravity is restricted to $\log g>3.5$ marked by the black dashed line. Further limits are applied to the period $P_{1}$ setting $0.5 \mathrm{~d} \leq P_{1} \leq 45 \mathrm{~d}$. The lower limit should exclude pulsations basically hitting on short time scales. The upper limit is approx. equal to half the time span of Q3. Since Kepler suffers from instrumental effects visible on timescales of the quarter duration periods longer than 45 days remain doubtful. We cannot distinguish between long-term variability and trends caused by the instrument since the periods are selected automatically by our algorithm. There exists a certain fraction of stars with long periods nonetheless. We cannot be sure that some of them are also due to instrumental effects, so they should be treated with some caution. Furthermore, all derived periods $P_{1}$ for relevant stars are compared to the orbital periods $P_{\text {orb }}$ from the lists of eclipsing binaries 5 and false positive 6 . If the relative difference $\left|P_{1}-P_{\text {orb }}\right| / P_{\text {orb }}<0.05$ these periods are discarded. This limit excludes orbital periods but we might lose some tidally locked systems.

\footnotetext{
${ }^{5}$ http://archive.stsci.edu/kepler/eclipsing_binaries.html

${ }^{6}$ http://archive.stsci.edu/kepler/false_positives.html
} 


\section{Limits \& Examples}

In this chapter we apply different filters to all stars that survived the upper limits to make sure that rotation is their favorite explanation. Finally, we show three examples where DR is the favorite explanation for the light curve pattern.

\subsection{Zero crossings}

After setting several constraints to $P_{1}$ we apply a filter to achieve periods originating from rotating active regions by counting the number of zero crossings of each light curve. For many possible realizations of a spotted star the light curve shows a sine-like variations with a defined number of zero crossings. Thus, a low number of crossing events is indicative for regular rotationinduced variations whereas a high number of zero crossings is considered as a hint for stellar pulsations and irregular variations. In this way we filter out periods that does not originate from rotation.

A single sine wave has two zero crossings per period. Thus, the number of zero crossings in Q3 equals $N_{\text {zero }}=90 \cdot 2 / P_{1}=180 f_{1}$ for a sine wave with a period $P_{1}$. To calculate $N_{\text {zero }}$ the light curves have to be smoothed. Since we are facing a variety of different rotation time scales (0.5-45 days) we cannot apply the same smoothing width to all light curves. The very fast rotators can only be smoothed on a few hours to stay below the rotation period. For the very slow rotators other effects (like e.g. granulation) become dominant on these time scales so the smoothing time needs to be sufficiently longer. To account for this effect we smooth the light curves over timescales proportional to their period $P_{1}$. Since Kepler long cadence data consists of $\sim 30$ minutes integrations we have $48 \cdot P_{1}$ data points in a certain period $P_{1}$. We smooth the data using a boxcar average with a width of $4 \cdot P_{1}$ data points which turns out to be a suitable width for fast and slow rotators. We plot the frequency $f_{1}$ vs. the number of zero crossings in Q3 in Fig. 7.1. The lower red line is equal to $N_{\text {zero }}=180 \cdot f_{1}$ as predicted for sine like variations. The upper one equals $2 N_{z e r o}=90 \cdot 4 / P_{1}$. This upper limit accounts for the cases of two active longitudes located on opposite sides of the star which produces up to 4 zero crossings per period. This trend is broken towards longer periods; the variations become irregular showing more zero crossings. A simple explanation is that the smoothing width is chosen too small. Another reason could be that star spots evolve on these long time scales $\left(P_{1}>20\right.$ days $)$ as seen on the sun. Stars with too many zero crossings are considered as irregular, quiet, pulsators, or giants with poor determined $\log g$. All stars lying above the upper red line (gray symbols) are discarded.

\subsection{Detection limit of $\alpha$}

After filtering out periods by their number of crossing events we focus on the cases where two periods have been found. The reliability of a second period strongly depends on the separation of the periods $P_{1}$ and $P_{2}$ in the periodogram. If they lie very close it's hard to tell whether the small separation comes from a very small $\alpha$ or if it's due to artifacts from our method. After visual inspection of several light curves we find that the typical separation of the two periods should be larger than 10 points on the frequency grid in the periodograms. Thus, we invert the periods to achieve two frequencies $f_{1}=1 / P_{1}$ and $f_{2}=1 / P_{2}$. If their absolute separation is less 


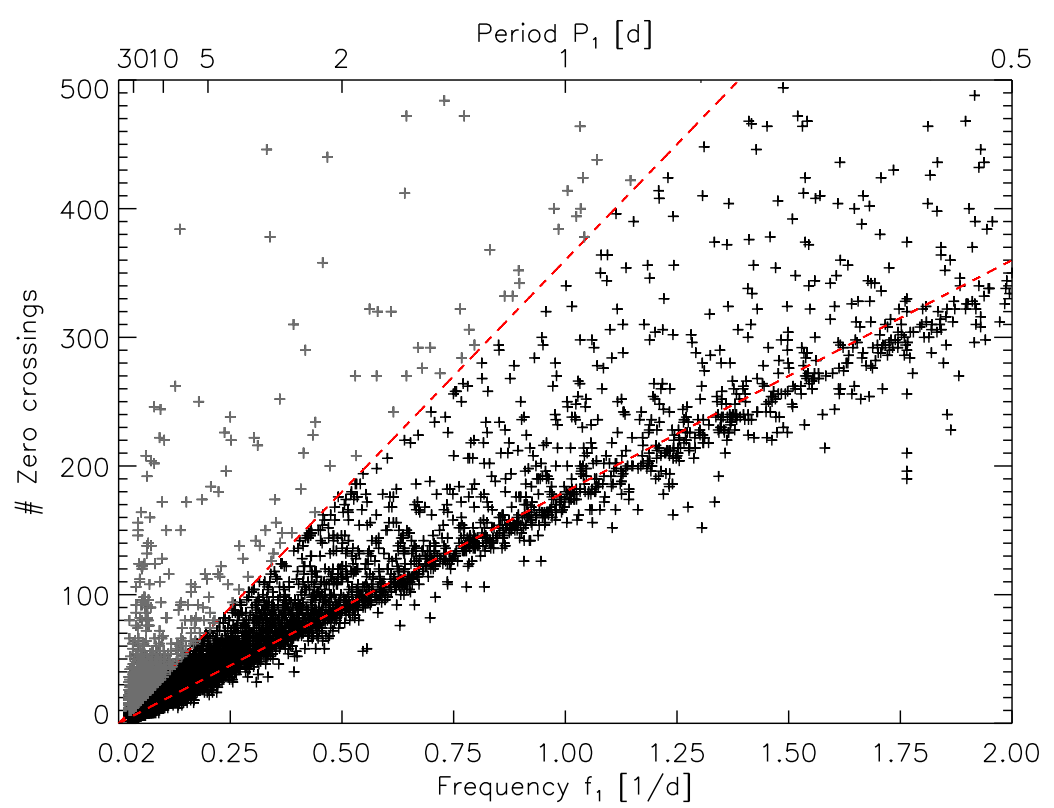

Figure 7.1.: Frequency $f_{1}$ vs. number of zero crossings in Q3. The lower red line equals the number of zero crossings expected for sine-like variations. Stars lying above the upper red line (gray symbols) are discarded since they show more than twice zero crossings as expected.

than 10 times the lowest frequency $f_{\text {low }}$ (sec. 6.1), i.e. $\left|f_{1}-f_{2}\right|<10 f_{\text {low }} \approx 0.0056 \mathrm{cyc} / \mathrm{d}$ then the second period is discarded.

For each of the above limits imposed upon the set of periods we might lose real rotation periods. This is not preventable since the analysis procedure is done automatically. All filters applied have decreased the number of false positives significantly yielding a condensed and reliable set of rotation periods. We find that 24.124 stars survive all filters, i.e. they have a measured rotation period $P_{1}$. For 18.619 stars a second period $P_{2}$ has been found. The following section shows example light curves, and the fits achieved by our method, and the associated periodograms. It demonstrates the problem of determining a second period from the periodograms, and shows the importance of pre-whitening.

\subsection{Examples: Light curves, Periodograms, and Rotation Periods}

Here, we briefly address the problem of associating the returned periods from the pre-whitening to rotation periods of active regions on the star. Fig. 7.2 shows an example of a fast rotator, and all five periodograms from the pre-whitening. The upper panel shows the light curve and the global sine fit of all five periods in red. The light curve shows a regular beating pattern most probably due to several active regions. The lower panel shows - from top to bottom - the first to the fifth periodogram.

The first periodogram reveals several distinct peaks close to the most significant one at 3.24 days which has been chosen as primary period $P_{1}$. In the second panel the periodogram of the residuals is taken. One clearly sees that the power around 3.24 days has basically dropped to zero whereas the second and third highest peak from the initial periodogram now exhibit the highest powers. The period with the second highest power around $P_{2}=3.57$ days has been chosen. From the third to the fifth panel we see that there are more periods close to $P_{1}$ probably due to other active regions.

In Fig. 7.3 we show the slow rotator KIC 1869783 and the associated periodograms. The panels 
are the same as in the previous plot. The light curve has a double-dip shape due to active regions located on opposite sides of the star. The initial periodogram shows a rather broad peak at 26.2 days which has been chosen as primary period $P_{1}$. The second periodogram shows a peak around 13.1 days which belongs the second active region on the opposite side of the star. From the light curve we can see how this region becomes shallower and the primary region more pronounced. The third periodogram has the highest peak around 68.5 days which belongs to a long-term behavior most probably due to the data reduction. The fourth periodogram shows a strong peak around 34.2 days that has unfortunately been discarded by the selection process because it lies beyond $30 \%$ of $P_{1}$. Only in the fifth pre-whitening step another period at 20.9 days has been found which was chosen as $P_{2}$ because it lies within $30 \%$ of $P_{1}$.

These two examples demonstrate the process used to detect the most significant periods. The beating pattern seen in the light curve in Fig. 7.2 is no proof of DR but that is the most probable explanation. The slow rotator shows active regions at different longitudes evolving with time. In this case it is not clear whether this is due to DR or spot growth or decay. Both figures demonstrate that the fits to the light curves reproduce the main activity pattern. They could be improved by using more than 5 sine waves but this would not change the two most significant periods. Furthermore, they point out the presence of a second period, and the difficulty to assign a physical meaning to them. 
KIC: 1995351
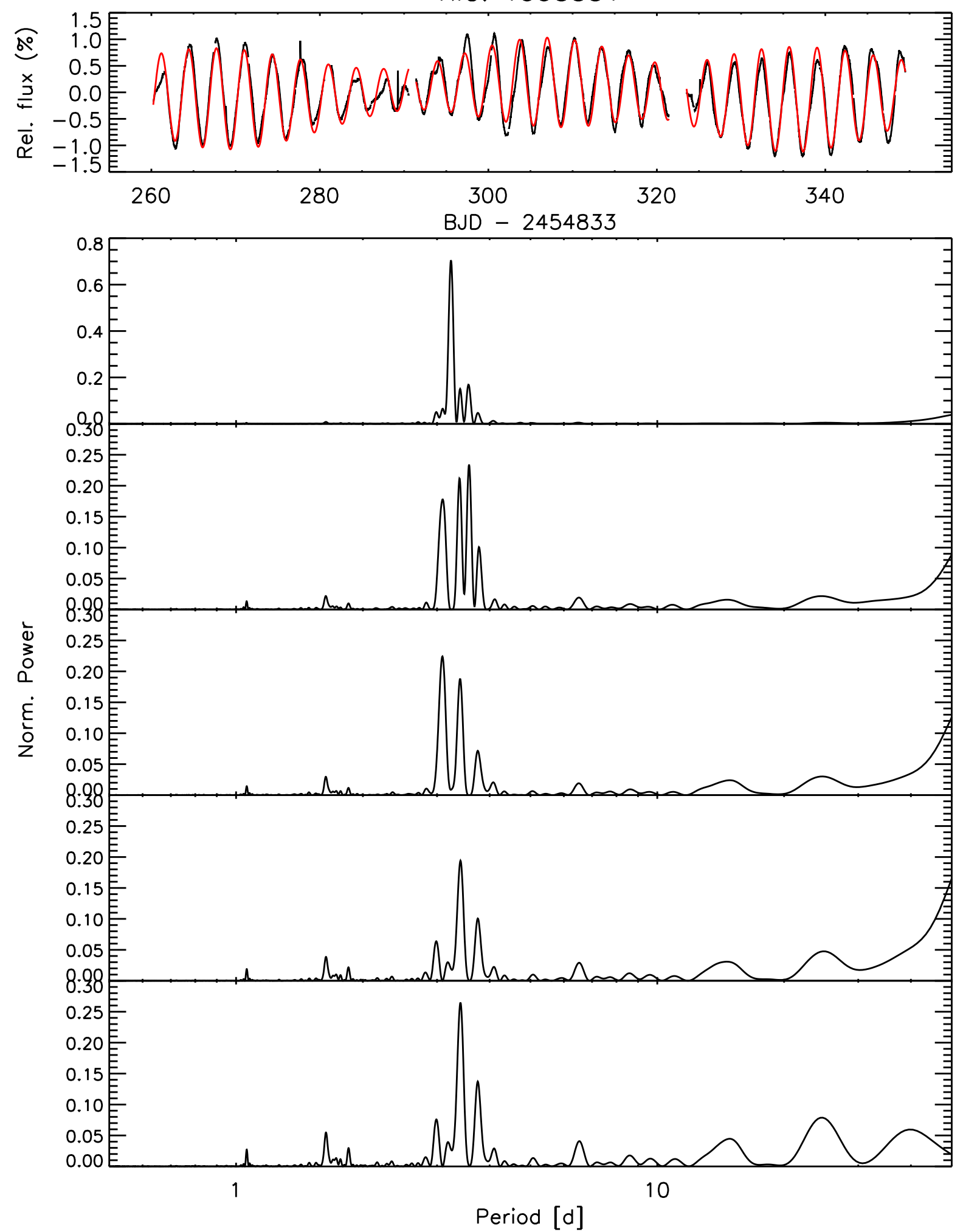

Figure 7.2.: Top Panel: Light curve \& global fit of the star KIC 1995351. Lower Panel (top to bottom): The first five periodograms from pre-whitening. The periods $P_{1}=3.24 d, P_{2}=3.57 d$ have been selected. 
KIC: 1995351
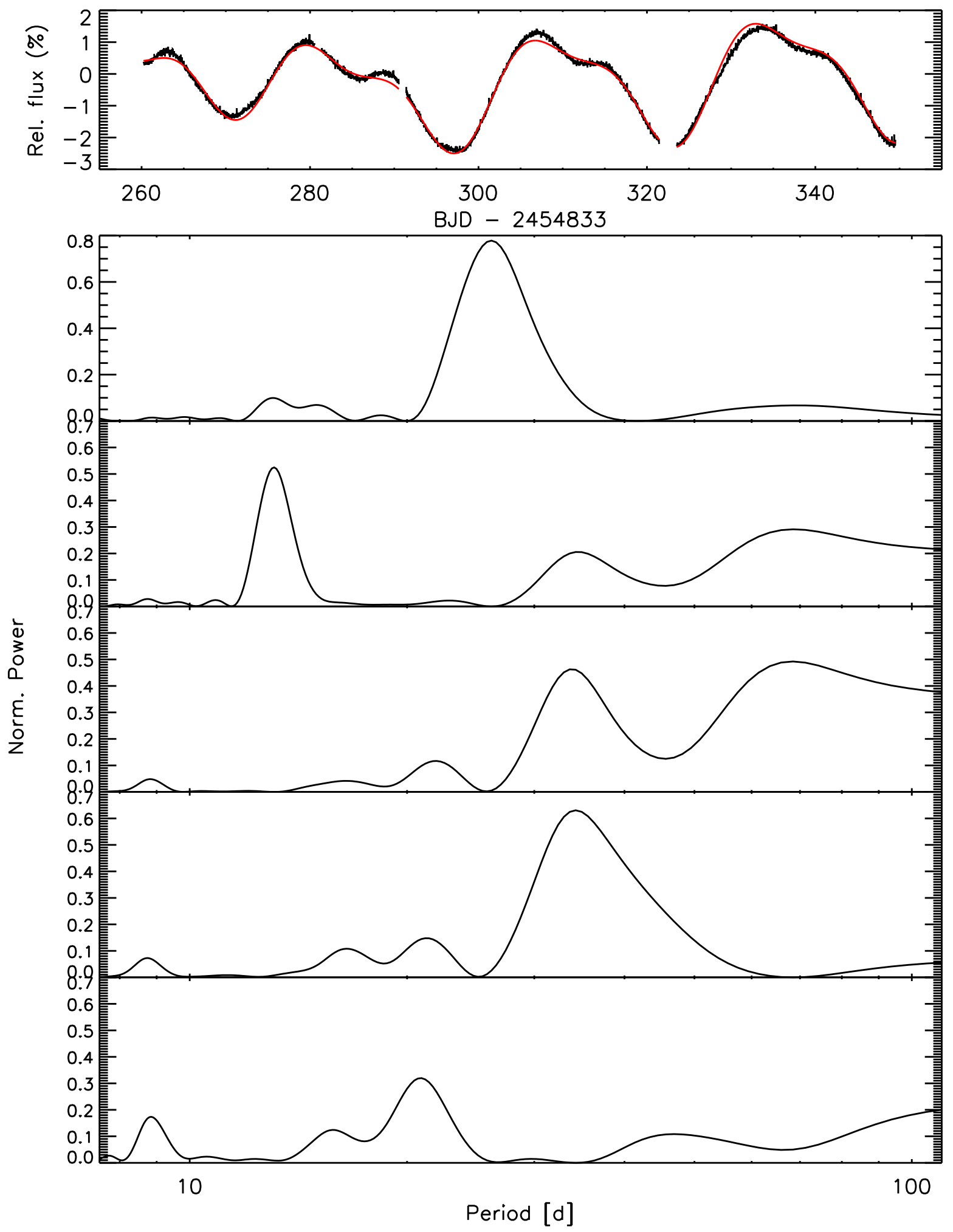

Figure 7.3.: Top Panel: Light curve \& global fit of the star KIC 1869783. Lower Panel (top to bottom): The first five periodograms from pre-whitening. The periods $P_{1}=26.2 d, P_{2}=20.9 d$ have been selected. 


\section{Kepler Results}

This chapter contains the results of the application of our method to the active Kepler stars providing rotation periods of more than 20.000 stars. Sec. 8.1 compares our rotation periods to previous measurements and sec. 8.2 shows that our periods are consistent with the concept of magnetic braking. The main focus lies on the detection of DR which is discussed in terms of relative and absolute shear in sec. 8.3 and 8.4. resp.. The number of false positives, i.e. those periods mis-classified as rotation, is estimated in sec. 8.5. Finally, in sec. 8.6] DR beyond $\alpha=0.3$ is considered.

\subsection{Rotation Periods}

In Fig. 8.1 we show the distribution of the rotation periods $P_{1}$ and $P_{2}$. We find mean values $\left\langle P_{1}\right\rangle=16.3 \mathrm{~d}$ and $\left\langle P_{2}\right\rangle=13.3 \mathrm{~d}$, with a spread of $\sigma\left(P_{1}\right)=10.1 \mathrm{~d}$ and $\sigma\left(P_{2}\right)=7.3 \mathrm{~d}$, respectively. The distribution of $P_{1}$ slightly decreases towards longer periods and levels off around $\sim 35$ days. Towards shorter periods a second peak between 0.5 and 2 days appears. The distribution of $P_{2}$ falls off more rapidly towards longer periods. Most of the "missing" periods $P_{2}$ are greater than 20 days, so some of them might lie below our detection limit. Thus, the mean values of the distributions do not necessarily indicate that a second period is more likely be found for shorter periods.

The dearth of slow rotators in both distributions is due to several reasons. The determination of long periods requires stable active regions on the star. On the Sun the spot lifetimes are of the order of the rotation period hampering the period detection. This does not need to be true for other stars though. Furthermore, long-term stellar variability and instrumental trends are currently difficult to distinguish in Kepler data. Both effects bias the distribution towards shorter periods. Our results apply primarily to periods shorter than about 30 days. Since we only analyzed one quarter so far, and applied an upper limit of 45 days, the distribution of slow rotators is not addressed in this study.

\subsection{Rotational Braking}

It is well-known that stellar rotation rates correlate with spectral type. Stars around spectral type $\mathrm{F}$ and earlier are known to be fast rotators. The convection zone grows towards cooler stars, and a dynamo mechanism generates magnetic fields. Ionized material follows the magnetic field lines (stellar wind) and carries away angular momentum resulting in a spin-down of the star (Barnes 2003; Reiners \& Mohanty 2012). This process is known as rotational braking, for which we find evidence in Fig. 8.2. We plot our most significant period $P_{1}$ against $B-V$ for 24.124 stars with at least one detected period (gray dots). Fig. 8.2 is a composition of previous rotation studies and our results. Filled circles represent data from Baliunas et al. (1996) (olive), Kiraga \& Stepien (2007) (purple), and Irwin et al. (2011) (red). Recently, rotation periods for the Kepler M dwarfs sub-sample have been published by McQuillan et al. (2013) (blue circles). Gray dots represent our measurements. For some of the other measurements no $B-V$ information was available. We transformed stellar masses into effective temperature using 1 Gyr isochrone models from Baraffe et al. (1998). The temperatures have been transformed into 


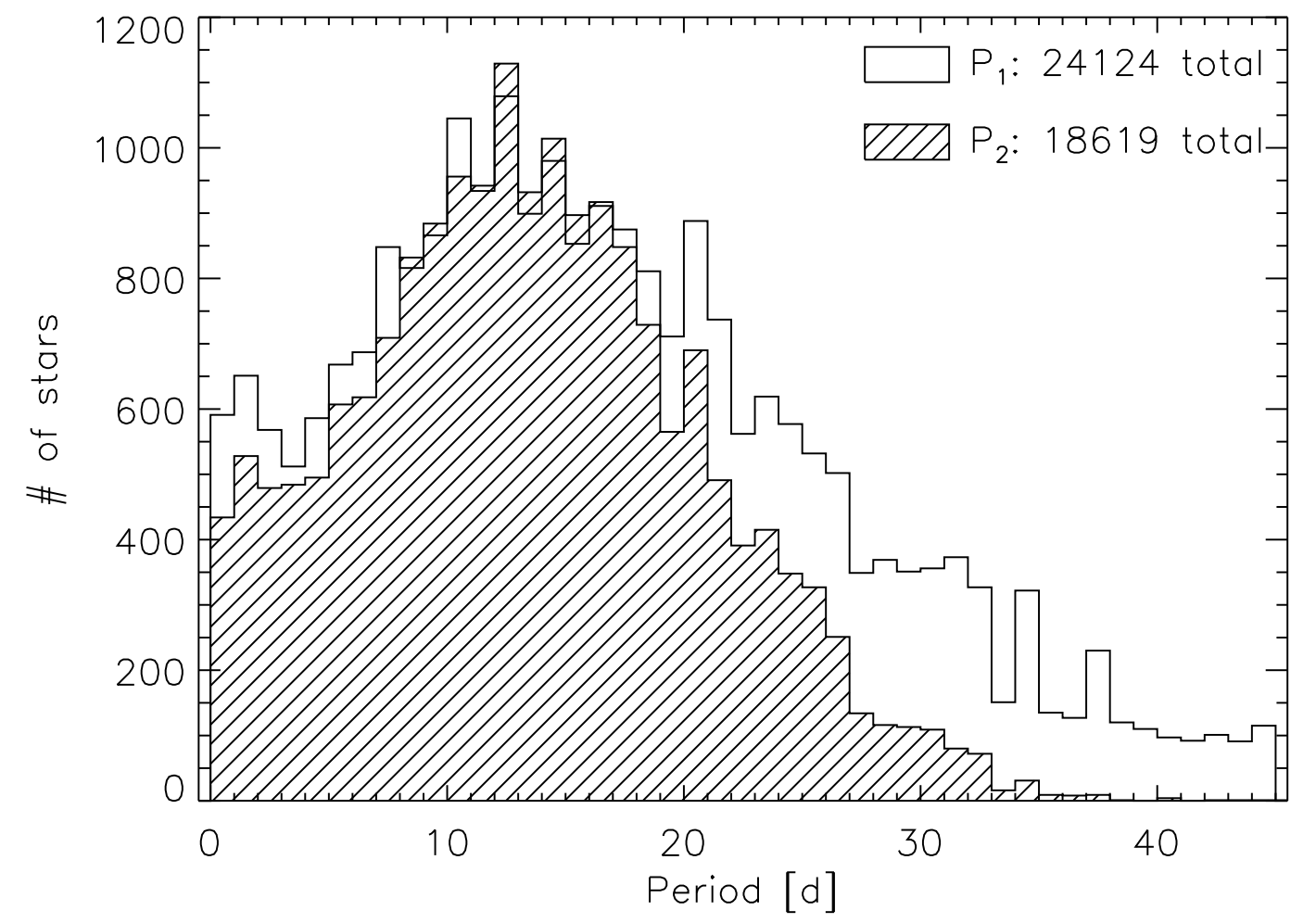

Figure 8.1.: Distribution of rotation periods $P_{1}$ and $P_{2}$. For 24.124 stars a rotation period $P_{1}$ could be detected; 18.619 of those exhibit a second period $P_{2}$.

$B-V$ using the relation from Reed (1998). For the Kepler stars we used the relation between $(g-r)$ and $B-V$ from Jester et al. (2005). The periods from Baliunas et al. (1996) form an upper envelope to our results. The results for the Kepler $M$ dwarfs (blue circles) show good agreement with our results although McQuillan et al. (2013) used an auto-correlation method which is a completely different mathematical tool.

Fig. 8.2 is consistent with the picture of rotational braking. A steep rise in rotation periods appears around $B-V=0.6$. In this region the convection zone starts to form and grows deeper in cooler stars. Thus, magnetic braking becomes stronger leading to a spin-down of the stellar rotation rate. Barnes (2007) empirically found a relation between $B-V$, age $t$, and rotation period

$$
P(B-V, t)=0.7725(B-V-0.4)^{0.601} t^{0.5189} .
$$

The age-dependence is similar to the classical Skumanich law $P \sim \sqrt{t}$ (see also Reiners \& Mohanty (2012)). The black curves in Fig. 8.2 represent isochrones with ages of 100, 600, 2000, and 4500 Myr. The distribution of periods in our sample follows a similar behavior as the isochrones indicating that stars with different color follow similar age distributions. Stars with rotation periods around 5 days are probably very young stars that did not have the time to spin-down. In Fig. 8.3, we show rotation period $P_{1}$ vs. range in a density plot. The bright region in the middle represents a high density whereas dark regions express low density. Young stars are not only expected to rotate fast but also to be very active. We find that the range increases with rotation supporting this relation between our measured rotation period and variability range. Hence, the variability range is a useful age and activity indicator.

We estimate the ages from rotational period for the Kepler stars using eq. (8.1). The distri- 


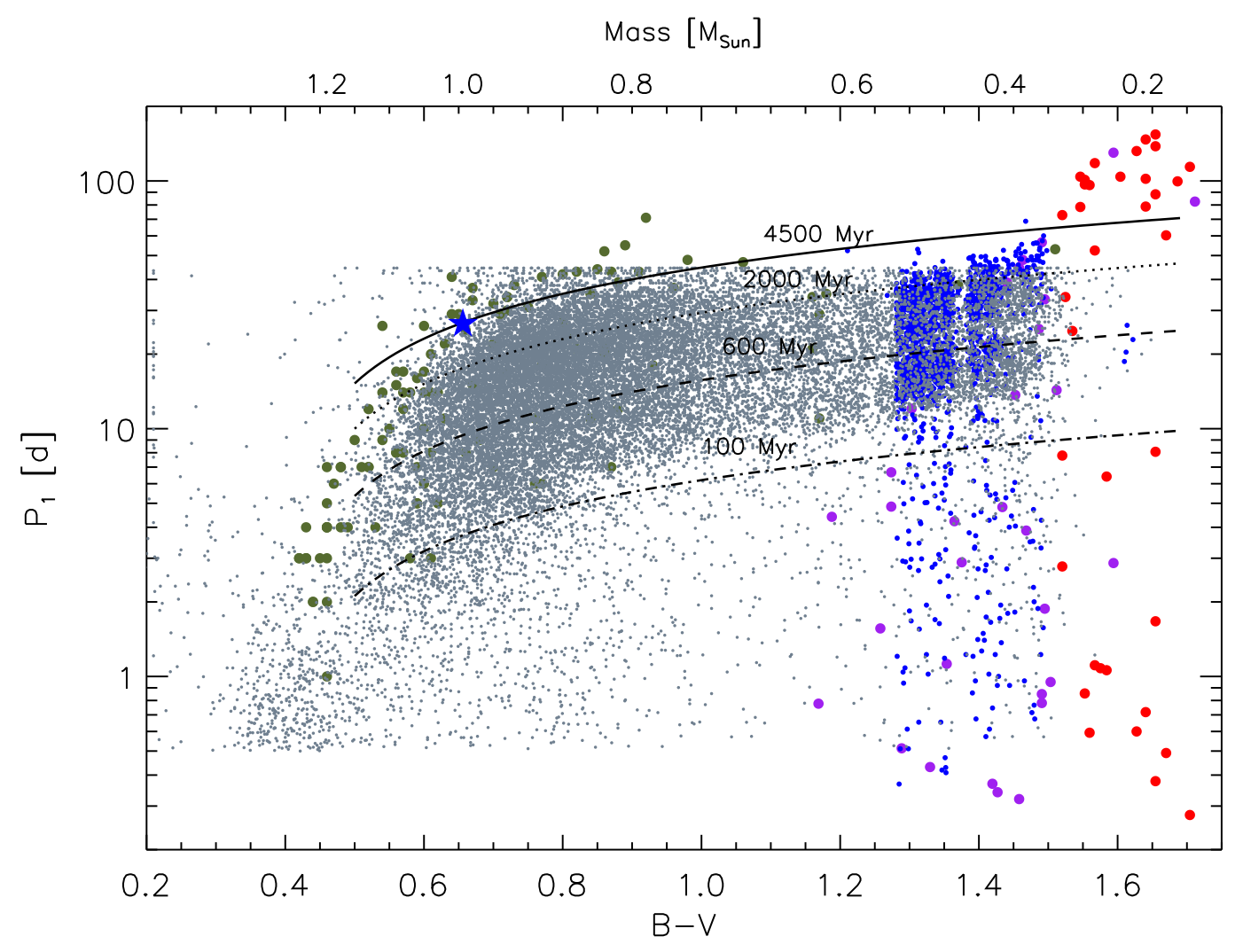

Figure 8.2.: $B-V$ vs. rotation period $P_{1}$ of 24.124 stars with at least one detected period (gray dots). The filled circles represent data from Baliunas et al. (1996) (olive), Kiraga \& Stepien (2007) (purple), Irwin et al. (2011) (red), and McQuillan et al. (2013) (blue). Towards cooler stars we see an increase in rotation periods with a steep rise around $B-V=0.6$ supplying evidence of rotational breaking. The black lines represent a color-period relation found by Barnes (2007) for different isochrones. The blue star represents the sun for comparison.

bution of ages in the active Kepler stars is shown in Fig. 8.4. The black histogram shows all stars with $0.5<B-V<1.0$, the red one covers $1.0<B-V<1.4$, and the blue one shows $1.4<B-V<1.5$. The steep decrease on the right-hand side of all three distributions can be understood by the missing long-period stars caused by our upper limit of 45 days and by our selection of active stars only. The left-hand side of the age-distribution is expected to be rather complete because the lower limit of detectable periods of 0.5 days is not relevant even for very young active stars. The dashed black line shows the distribution of stars according to a uniform distribution of stellar ages (plotted on a logarithmic scale). The dashed curve is remarkably similar to the left-hand side of the black distribution up to an age of $\sim 300 \mathrm{Myr}$.

McQuillan et al. (2013) found evidence for a bimodal period distribution in the Kepler M dwarf sample $(1.21<B-V<1.62)$. The bimodality is explained by the existence of two distinct stellar populations. This gap at $P_{1} \sim 30$ days also appears in our data around $1.4<B-V<1.5$ and corresponds to an age of roughly $800-900 \mathrm{Myr}$. We find a similar feature in hotter stars $(1.0<B-V<1.4)$ at $P_{1} \sim 20 \mathrm{~d}$. This feature, however, corresponds to a significantly younger age of $600-800 \mathrm{Myr}$ and is unlikely to be caused by the same age distribution as the gap in cooler stars. Whether the two period gaps are caused by a selection bias affecting our period sample or by a predominance of certain ages in the distribution of stellar ages (potentially introduced by stellar clusters in the Kepler field, see e.g. Meibom et al. (2011)) needs to be further tested. Furthermore, in comparison to a constant star formation history (dashed red line in Fig. 8.4), 


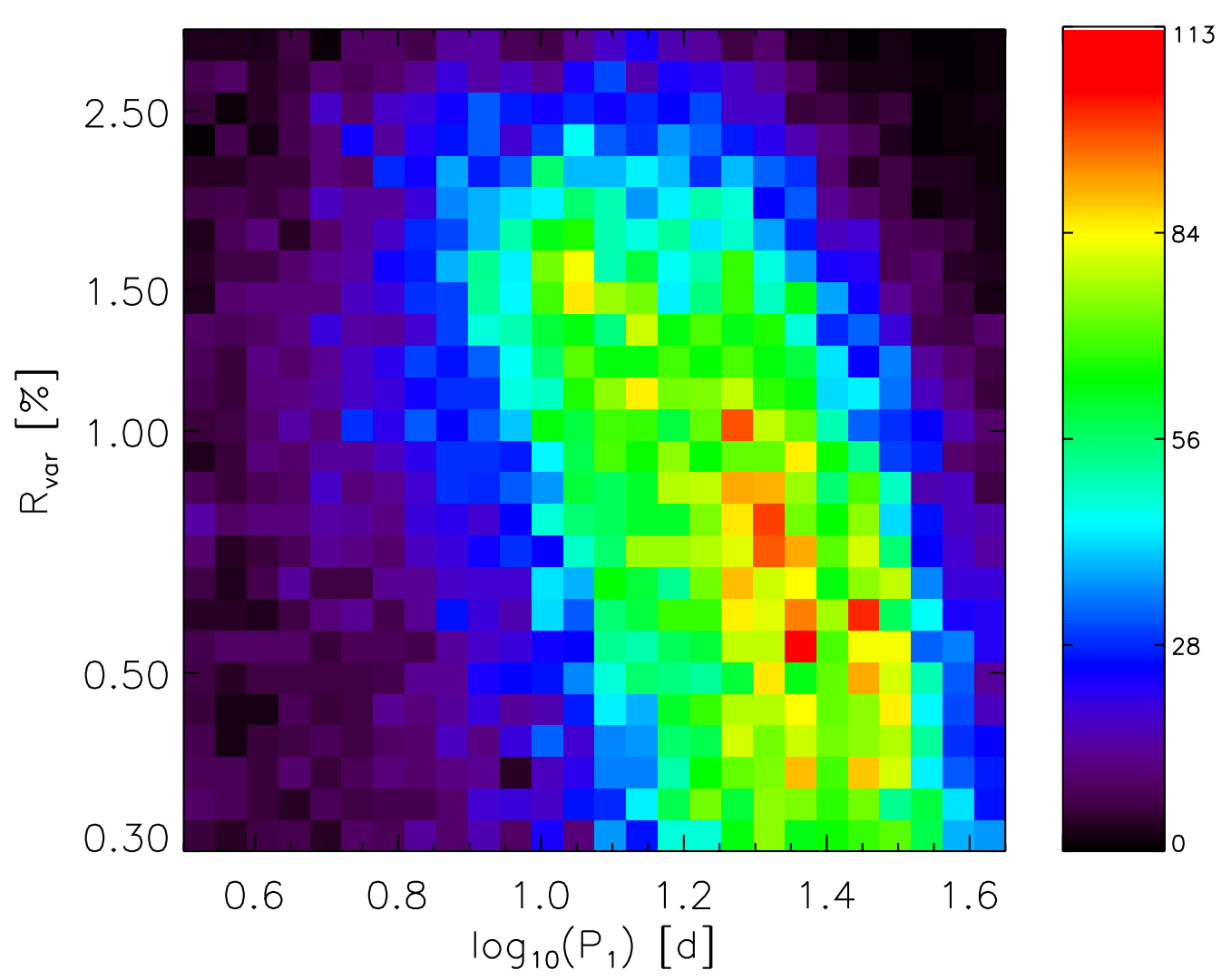

Figure 8.3.: Density plot of rotation period $P_{1}$ vs. $R_{v a r}$ with bright region representing a high density whereas dark regions express low density. The annotation of the color bar contains the total number of stars in each bin. The distribution shows that the range increases towards shorter periods. Since fast rotators are expected to exhibit an enhanced level of activity the range could probably be used as proxy for stellar activity.

the sample with $1.0<B-V<1.4$ (red histogram) lacks stars younger than $\sim 200 \mathrm{Myr}$. The dearth of young objects as well as the gaps in the age distributions cannot be easily explained and need further investigation.

\subsection{Relative Differential Rotation $\alpha$}

In Fig. 8.5 we plot the relative DR $\alpha$ against the minimum rotation period $P_{\min }$. We find that $\alpha$ increases with rotation period. The black dashed line marks the detection limit (sec. 7.2) of our method. If we sort both periods that $P_{1}=P_{\min }$ and $P_{2}=P_{\max }$ then the absence of data points below this line can be understood by considering the relation

$$
\begin{gathered}
\left|P_{1}-P_{2}\right|=\frac{\left|f_{1}-f_{2}\right|}{f_{1} f_{2}} \geq 10 f_{\text {low }} P_{1} P_{2} \\
\Rightarrow \quad \alpha=\frac{\left|P_{1}-P_{2}\right|}{P_{\text {max }}} \geq 10 f_{\text {low }} P_{\text {min }}
\end{gathered}
$$

Thus, all data points lie above the black line, it being the lower limit for $\alpha$. It is worth noting that more than $75 \%$ of all stars with detected periods lie above the detection limit since 18.619 of 


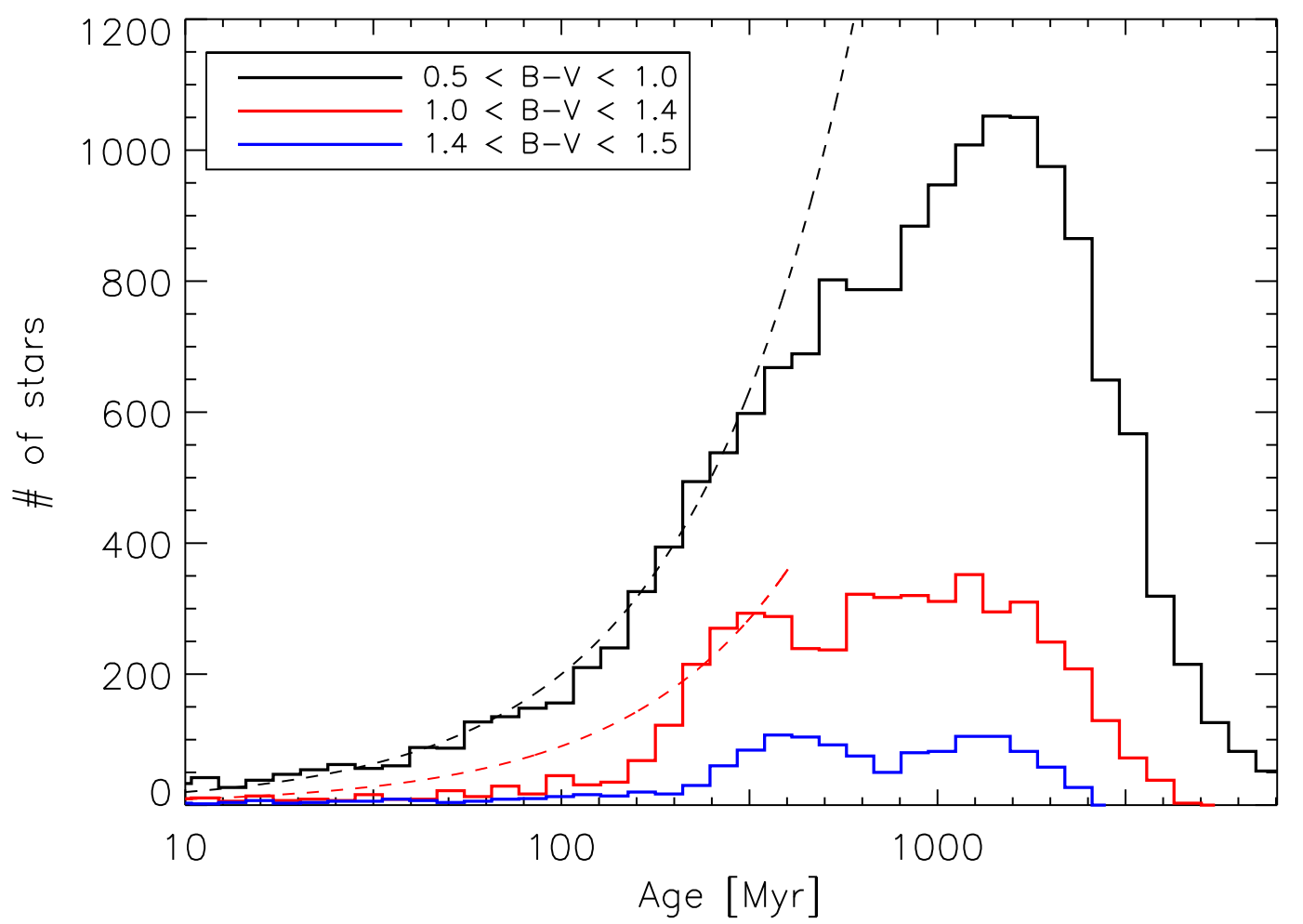

Figure 8.4.: Distribution of ages inferred from rotation periods $P_{1}$ for different color bins. For $B-V>1.0$ (red and blue) we see a bimodal distribution in agreement with McQuillan et al. (2013). The dashed black and red lines mark uniform age distributions.

24.124 stars exhibit a second period. In other words, only the 5505 stars where only one period has been detected can either lie below the detection limit, or above (below) our $\alpha_{\max }\left(\alpha_{\min }\right)$ values, respectively. In sec. 8.6 we discuss different $\alpha_{\max }$ values, and show that there are periods that have been discarded here due to the imposed $30 \%$ limit. Lowering $\alpha_{\min }$ does not yield much different periods, so we conclude that the general trend of increasing $\alpha$ with rotation period is not biased by our detection limit.

The colors in Fig. 8.5 represent different temperature bins. Towards cooler stars $\left(T_{\text {eff }}<5000 \mathrm{~K}\right.$, red and purple dots) the rotation period increases, confirming the result from Fig. 8.2. Hot stars above $6000 \mathrm{~K}$ (green and black dots) mostly populate the short periods covering the whole $\alpha$ range.

In Fig. 8.6 we show temperature vs. $\alpha$ correlating our results with the variability range. The colors indicate different ranges growing from $0.3 \%$ (yellow) to high ranges with amplitudes above $5 \%$ (purple). A shallow trend towards higher $\alpha$ with decreasing temperature is visible. A correlation with range can only be found for stars with very high ranges (purple dots). These stars mostly cover the region with $\alpha \lesssim 0.05$ over a large temperature range. If we think of the variability range as activity indicator this might confirm the hypothesis that these stars are very young since a low DR $\alpha$ indicates short periods. 


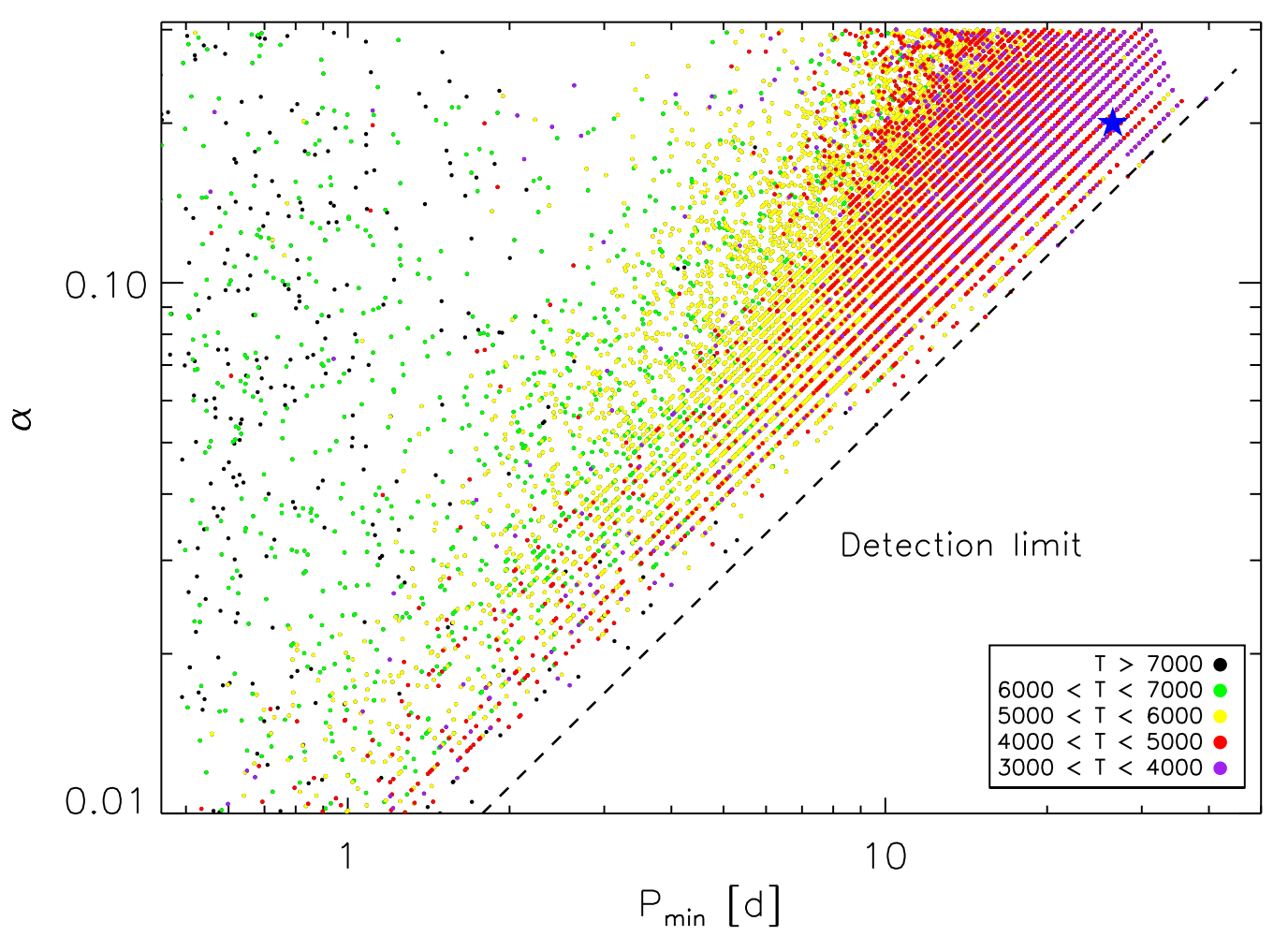

Figure 8.5.: Rotation period $P_{\min }$ vs. $\alpha$ for all stars with 2 detected periods. The relative shear $\alpha$ grows towards longer rotation periods. The colors represent different temperature bins, the dashed black line marks the detection limit, and the blue star shows the sun for comparison.

\subsection{Absolute horizontal shear $\Delta \Omega$}

We define the absolute horizontal shear as

$$
\Delta \Omega:=2 \pi\left|f_{1}-f_{2}\right|
$$

In Fig. 8.7 we plot $\Delta \Omega$ against the rotation period $P_{\min }$. For periods longer than 2 days $\Delta \Omega$ shows weak dependence on rotation period. Towards shorter periods (less than 2 days) $\Delta \Omega$ slightly increases showing a large spread of the order of one magnitude. The detection limit is marked by the horizontal dashed line. The diagonal dashed lines mark the upper and lower limits, $\alpha_{\max }$ and $\alpha_{\min }$, resp., and the colors are the same as in Fig. 8.5.

The temperature dependence of $\Delta \Omega$ is shown in Fig. 8.8 with our measurements shown as gray dots. The olive diamonds and error bars are measurements taken from Barnes et al. (2005). The olive dashed line is the fit to these data taken from Collier Cameron (2007). The orange data and error bars are measurements from Ammler-von Eiff \& Reiners (2012). The red dash-dotted line and the light blue dashed line are fits to theoretical predictions from Küker \& Rüdiger (2011). The black dashed line shows the detection limit, and the blue star marks the solar value for comparison. In contrast to earlier studies (Barnes et al. 2005; Reiners 2006; Collier Cameron 2007; Ammler-von Eiff \& Reiners 2012) claiming strong dependence of $\Delta \Omega$ on temperature we find two temperature regions with different behavior of $\Delta \Omega$. We find only weak dependence of $\Delta \Omega$ with temperature over a large range from $3500-6000 \mathrm{~K}$. Above $6000 \mathrm{~K}$ the absolute shear $\Delta \Omega$ shows a steep increase with temperature although the data support no conclusion about the functional form of a fit. The fit from Barnes et al. (2005) (olive dashed line) does not provide 


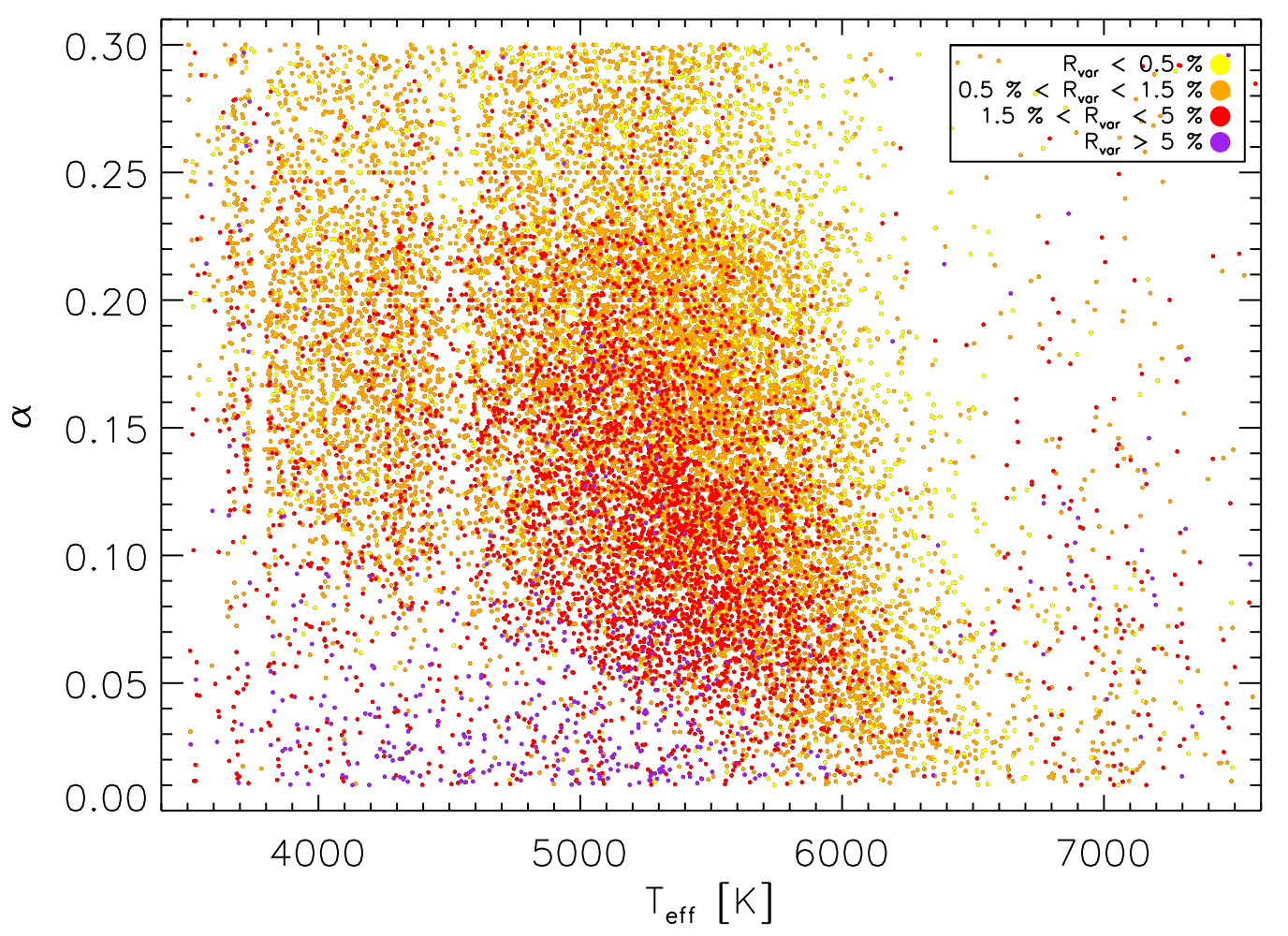

Figure 8.6.: Temperature vs. relative shear $\alpha$. We see that $\alpha$ slightly increases towards cooler stars. The colors indicate different variability ranges. A distinct correlation between $\alpha$ and the range is only visible for the stars with very high ranges $R_{v a r}>5 \%$. These stars group at small $\alpha$ values over a large temperature range probably indicating their young age.

a reasonable fit, but the data show good agreement with theoretical curves (red and light blue line) provided by Küker \& Rüdiger (2011). They also found that the temperature dependence of $\Delta \Omega$ cannot be represented by a single fit but requires two fits for distinct temperature regions (comp. Fig. 2 in Küker \& Rüdiger (2011)).

Küker \& Rüdiger (2011) predict a shallow increase in $\Delta \Omega$ for temperatures between 3500 and $6000 \mathrm{~K}$ (red dash-dotted line). Since this behavior is not evident for our measurements we calculated $\Delta \Omega$ histograms for temperature bins of $250 \mathrm{~K}$ between $3500 \mathrm{~K}$ and $6250 \mathrm{~K}$. The weighted means $\langle\Delta \Omega\rangle$ of the distributions are shown as open black circles in Fig. 8.8. The mean values slightly increase with temperature as predicted by Küker \& Rüdiger (2011). This shallow increase suggests good agreement between theory and observations but should be treated with caution. For the 5505 stars where only one period has been detected we cannot tell whether these stars exhibit a small shear $\Delta \Omega$ that lies below our detection limit, or if a second period has not been detected for other reasons. A fraction of these 5505 stars can change the trend so we cannot draw strong conclusions about the behavior below the limit of $\Delta \Omega \approx 0.035 \mathrm{rad} \mathrm{d}^{-1}$.

\subsection{False Positives}

In this section we statistically estimate the number of periods in our final sample that survived all filters but are probably not due to rotation. Other sources of periodic stellar variability are e.g. binarity, pulsations, or instrumental effects. We call these detections false positives (FPs). 


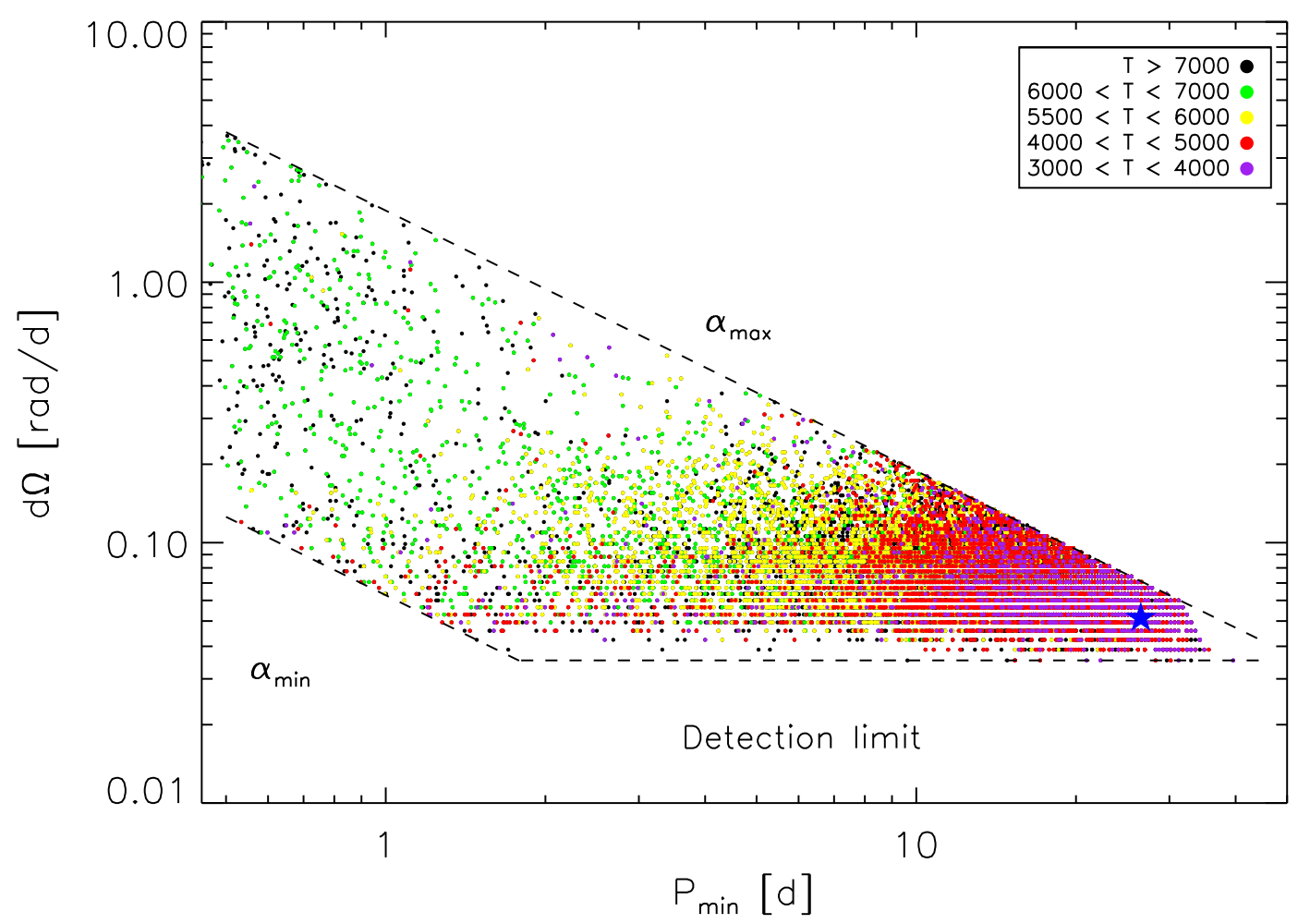

Figure 8.7.: Rotation period $P_{\min }$ vs. absolute shear $\Delta \Omega$. The shear $\Delta \Omega$ shows weak dependence on rotation period for $P_{\min }>2 \mathrm{~d}$, and increases towards fast rotators showing a large spread. The colors are the same as in Fig. 8.5, the dashed lines show the detection limit, and the upper and lower limits $\alpha_{\max }$ and $\alpha_{\min }$, resp.. The blue star shows the sun for comparison.

We define three classes of rotational variability: the rapid rotators $(0.5 d<P<10 d)$, the moderate rotators $(10 d<P<20 d)$, and the slow rotators $(P>20 d)$. Example light curves representative for the first and last group are shown in Fig. 7.2 and 7.3 . Since the sample is too large to inspect each light curve individually we randomly selected 100 stars of each rotation class and checked their light curves by eye. This will only give a rough error estimate because only a small number of stars is inspected, and the method is very subjective.

Orbital periods of eclipsing binaries or transiting planets should be relatively rare in the final sample. The eclipsing binary list ${ }^{5}$ has been cross-matched with our sample and stars with coinciding KIC numbers have been discarded. For planets or planetary candidates the returned periods are most dominantly due to stellar activity. The transits have very little data points compared to the whole light curve, and the periodogram is not very sensitive to these periods. We didn't find any period associated to eclipsing binaries or planetary transits.

In sec. 6.2 we set a lower limit of 0.5 days to our periods since pulsations mostly occur on timescales less than half a day (e.g. $\delta$ Scuti stars). There do exist $\gamma$ Doradus stars showing periods and light curve shapes similar to spot-induced variability in the range of $0.5-4$ days (Balona et al. 2011b). From the light curve alone it is challenging to distinguish between rotation and pulsation in this regime (Debosscher et al. 2011). Hence, the short period regime should be treated with caution. The rapid rotators class exhibits 6 stars showing irregular variations which could be a hint for stellar pulsations, 6 alias periods, and 2 periods without any reference to the light curve.

For the moderate rotators class alias periods are the largest error source. In 6 cases the detected 


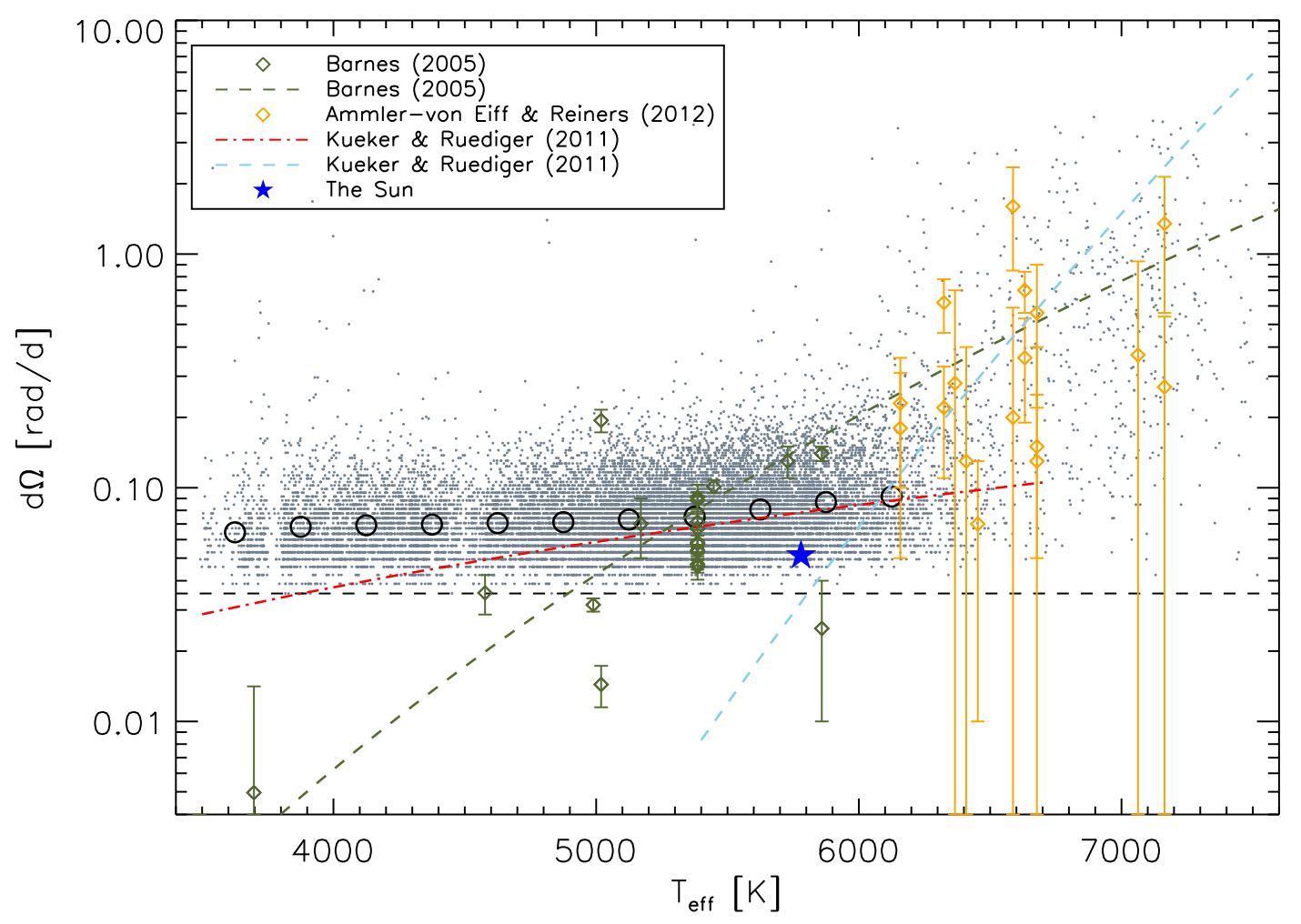

Figure 8.8.: Temperature vs. horizontal shear $\Delta \Omega$ of our data (gray dots) compared to previous measurements and theoretical results: Barnes et al. (2005) (olive diamonds), Ammler-von Eiff \& Reiners (2012) (orange diamonds), Küker \& Rüdiger (2011) (red and light blue curves). The black circles denote mean values of our measurements for different temperature bins. The black dashed line shows the detection limit, and the blue star marks the solar value for comparison. The figure shows that $\Delta \Omega$ only weakly depends on temperature over a large range from $3500-6000 \mathrm{~K}$. Above $6000 \mathrm{~K}$ the shear strongly increases following a relation similar to Barnes et al. (2005) but with large scatter.

period is most probably the half of the true rotation period, and in one case the determined period could not be confirmed by visual inspection of the light curve.

The slow rotators class with periods greater than 20 days mostly suffers from instrumental effects. After each third of a quarter $(\sim 30$ days $)$ data is down-linked to earth cutting the quarter into three segments. Each of them can show individual trends so on time scales larger than 30 days the accuracy of the derived periods strongly depends on the data reduction pipeline. Furthermore, we expect that star spots may evolve on the time scales as seen on the Sun for stars with solar rotation periods, which distorts the light curve shape and makes it more difficult to detect stable periods. In this class only one alias period was detected, but 12 stars that didn't show clear counterparts to the rotation period in the light curve (like regular dips or double dips as seen for stars rotating faster).

In total 300 stars have been inspected. Summarizing results for the above rotation classes we find that about $12 \%$ of all their periods should probably be attributed to sources of periodic variability other than rotation. In the next section we discuss relative DR beyond $\alpha=0.3$. 
Table 8.1.: Number of stars with second period found for different $\alpha_{\max }$ values.

\begin{tabular}{cc}
$\alpha_{\max }[\%]$ & 2nd period found \\
\hline 5 & 1966 \\
10 & 5437 \\
15 & 9511 \\
20 & 13355 \\
25 & 16426 \\
30 & 18619 \\
35 & 20379 \\
40 & 21559 \\
45 & 22319 \\
50 & 23206
\end{tabular}

\subsection{Differential Rotation beyond $\alpha=0.3$}

In relation 6.2 an upper limit of $\alpha_{\max }=0.30$ has been used while searching for a second period. In this section we demonstrate how different upper limits $\alpha_{\max }$ change the total number of detections and the overall behavior of $\alpha$ with temperature and rotation period. We use 10 equidistant values $0.05 \leq \alpha_{\max } \leq 0.50$ (comp. Table 8.1). With increasing $\alpha_{\max }$ we find a larger total number of periods. The case $\alpha_{\max }=0.5$ is shown to demonstrate the limits of our method. This will become evident in the next two figures. For all stars with two detected periods we plot their density in the $T_{\text {eff }}-\alpha$ plane in Fig. 8.9. The colors are the same as in Fig. 8.3 with bright regions representing a high density. For each $\alpha_{\max }$ value we find that $\alpha$ slightly increases towards cooler stars. The case with the lowest upper limit $\left(\alpha_{\max }=0.05\right)$ looks a bit scattered but not very different than the general trend. The plot in the lower right corner $\left(\alpha_{\max }=0.5\right)$ demonstrates the limits of our method. Many stars "jump" to the upper limit $\alpha=0.5$ because an alias period is chosen by the algorithm. The $T_{\text {eff }}$ values from the KIC are not very accurate and neither are the stellar radii. We find the same trend vs. $\alpha$ however, i.e. the $\alpha$ value increases towards smaller radii (although this may not be an independent constraint). Our previous result that $\alpha$ increases towards longer rotation periods holds for all $\alpha_{\max }$ values. In Fig. 8.10 we show the density in the $P_{1}-\alpha$ plane. We see that the density is localized in a sharp strip that smears out for limits greater than $\alpha_{\max }=0.3$. These large $\alpha$ values belong to periods $P_{2}$ much longer than 45 days where instrumental effects play a dominant role. Again, the stars "jump" to the upper limit in the $\alpha_{\max }=0.5$ case. In the next part we compare our results to observations and theory, summarize the main results, and provide a brief outlook of future plans. 

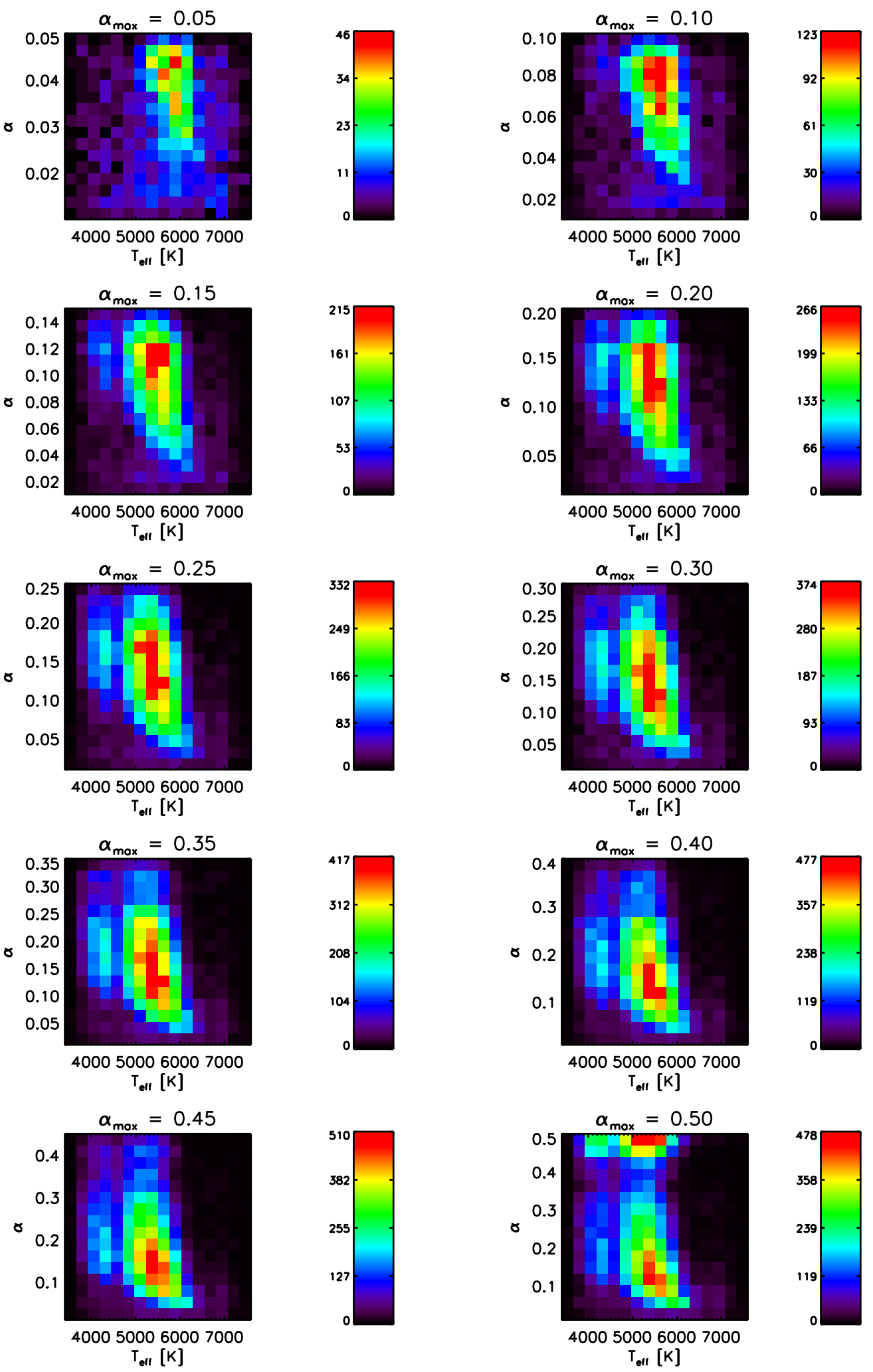

Figure 8.9.: Density plot in the $T_{\text {eff }}-\alpha$ plane for different values of $\alpha_{\max }$. For each $\alpha_{\max }$ value we find that $\alpha$ slightly increases towards cooler stars. The plot in the lower right corner $\left(\alpha_{\max }=0.5\right)$ demonstrates that our method is limited to $\alpha_{\max }<0.5$. Colors are the same as in Fig. 8.3. 

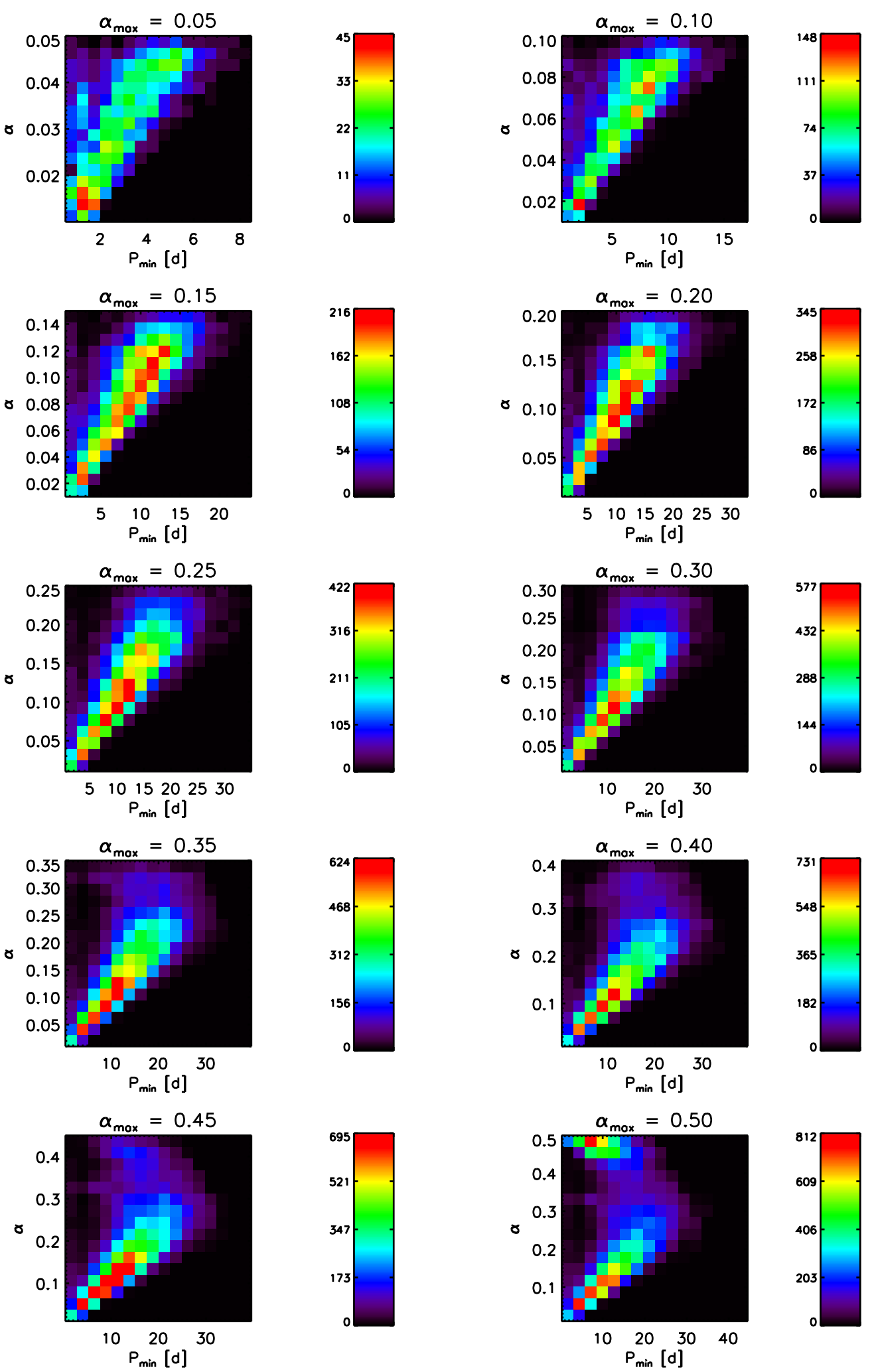

Figure 8.10.: Density plot in the $P_{1}-\alpha$ plane for different values of $\alpha_{\max }$. For each $\alpha_{\max }$ value we find that $\alpha$ strongly increases with rotation period. The plot in the lower right corner $\left(\alpha_{\max }=0.5\right)$ demonstrates that our method is limited to $\alpha_{\max }<0.5$. Colors are the same as in Fig. 8.3. 

Part IV.

Discussion 



\section{Comparison to other observations}

The rotation periods of the active Kepler stars are consistent with previous rotation measurements (Fig. 8.2), supporting the picture of stars losing angular momentum due to stellar winds that was deduced from a long history of observations. The situation for DR observations is different. In contrast to Barnes et al. (2005) we find that $\Delta \Omega$ weakly depends on temperature for the cool stars $(3000-6000 \mathrm{~K})$. Above $6000 \mathrm{~K} \Delta \Omega$ increases with temperature but the stars show no systematic trend but seem to be randomly distributed in this temperature regime. Using Doppler Imaging (DI) Barnes et al. (2005) found that the horizontal shear $\Delta \Omega$ strongly depends on effective temperature $\Delta \Omega \sim T_{\text {eff }}^{8.92}$. Five stars of their sample lie below our detection limit (Fig. 8.8). Collier Cameron (2007) combines results from DI and the Fourier transform method yielding the equation $\Delta \Omega=0.053\left(T_{\text {eff }} / 5130\right)^{8.6}$. The two groups we found (above and below $6000 \mathrm{~K}$ ) are also consistent with recent theoretical studies (see chapter 10).

The relation between rotation period and DR has been studied by several authors. Hall (1991) finds that the relative horizontal shear $\alpha$ increases towards longer rotation periods. Donahue et al. (1996) confirm this trend finding $\Delta P \sim\langle P\rangle^{1.3 \pm 0.1}$, independent of the stellar mass. Using the Fourier transform method Reiners \& Schmitt (2003) also find that $\alpha$ increases with rotation period for F-G stars. This result has been confirmed by Ammler-von Eiff \& Reiners (2012) compiling previous results and new measurements for A-F stars. Barnes et al. (2005) find that $\Delta \Omega$ only weakly correlates with rotation rate according to $\Delta \Omega \sim \Omega^{0.15}$.

Observations of DR cover a wide range of $\alpha$ values. The Doppler Imaging technique is particularly sensitive to small DR limited by the spot lifetimes, $\alpha \lesssim 0.01$ (see e.g. measurements by Donati \& Collier Cameron (1997) for AB Dor), although there are measurements (Donati et al. 2003) which yield $\alpha \approx 0.05$ for LQ Hya, and new measurements (Marsden et al. 2011) who find values between $0.005 \lesssim \alpha \lesssim 0.14$. The Fourier transform method (e.g. Reiners \& Schmitt (2003)) is sensitive to $\alpha>0.1$ and has been used to determine surface shears as large as $50 \%$ for some A-F stars. With our method we are able to detect DR up to $\alpha<0.5$.

In the following we compare our results with previous rotation measurements of individual Kepler stars (comp. Table 9.1). For three Kepler stars (KIC 8429280, KIC 7985370, KIC 7765135) DR has been measured (Frasca et al. 2011; Fröhlich et al. 2012) fitting an analytical spot model to the data. Their findings show good agreement to our results (see Table 9.1).

Several Kepler stars have been measured by Savanov using a light curve inversion technique that constructs a map of surface temperature. Savanov (2011b) considers the planet-candidate host stars KOI 877 and KOI 896 (KIC 7287995 and KIC 7825899, resp.) finding rotation period of 13.4 and 25.2 days, resp., stating that both stars exhibit active longitudes separated by about $180^{\circ}$. For KIC 7287995 we find $P_{1}=13.5 \mathrm{~d}$ and $P_{2}=10.4 \mathrm{~d}$, and for KIC $7825899 P_{1}=12.4 \mathrm{~d}$. In the latter case the detected period is an alias of the true rotation period due to the active longitudes.

The K dwarf KIC 8429280 is studied in Savanov (2011a) who finds brightness variations with periods of 1.16 and 1.21 days, consistent with the results from Frasca et al. (2011). In this case we find $P_{1}=1.16 \mathrm{~d}$ and $P_{2}=1.21 \mathrm{~d}$.

The fully convective M dwarf GJ 1243 (KIC 9726699) was studied in Savanov \& Dmitrienko (2011) yielding a rotation period of 0.593 days. Our algorithm detects a period of 118.7 days due to improper data reduction in Q3 but this period is filtered out by the upper limit of 45 days. The second strongest period we found was 0.59 days in agreement with Savanov \& Dmitrienko 
Table 9.1.: Comparison with previous rotation measurements for Kepler. References in the third column: (1) Frasca et al. (2011), (2) Fröhlich et al. (2012), (3) Savanov (2011b), (4) Savanov (2011a), (5) Savanov \& Dmitrienko (2011), (6) Savanov \& Dmitrienko (2012), (7) Bonomo \& Lanza (2012), (8) Roettenbacher et al. (2013).

\begin{tabular}{ccccc}
\hline KIC & $\begin{array}{c}\text { Period(s) } \\
{[\mathrm{d}]}\end{array}$ & Ref. & $\begin{array}{c}P_{1} \\
{[\mathrm{~d}]}\end{array}$ & $\begin{array}{c}P_{2} \\
{[\mathrm{~d}]}\end{array}$ \\
\hline 8429280 & $1.16-1.20$ & 1 & 1.16 & 1.21 \\
7985370 & $2.84-3.09$ & 2 & 2.84 & 3.09 \\
7765135 & $2.40-2.57$ & 2 & 2.55 & 2.40 \\
\hline 7287995 & 13.4 & 3 & 13.5 & 10.4 \\
7825899 & 25.2 & 3 & 12.4 & - \\
8429280 & $1.16,1.21$ & 4 & 1.16 & 1.21 \\
9726699 & 0.59 & 5 & - & - \\
2164791 & 3.36 & 6 & 3.35 & 3.27 \\
10619192 & 12.01 & 7 & 6.05 & - \\
5110407 & 3.47 & 8 & 3.61 & 3.42 \\
\hline
\end{tabular}

(2011). This period does not lie within $30 \%$ of the first one, and hence has not been chosen. Savanov \& Dmitrienko (2012) also study the fully convective, low-mass M dwarf LHS 6351 (KIC 2164791). They detect a rotation period of $3.36 \mathrm{~d}$, and evidence for DR in terms of $\Delta \Omega=0.006-0.014 \mathrm{rad} / \mathrm{d}$ from the evolution of surface temperature inhomogeneities. Their value lies below our detection limit; we find $P_{1}=3.35 \mathrm{~d}$ and $P_{2}=3.27$ d, i.e. $\Delta \Omega=0.046$. Visual inspection of the light curve in Q3 supports the larger value of $\Delta \Omega$ because a second active region appears after some time which is not seen in Q1-Q2 data. Unfortunately, this star misses in our final list because it has no effective temperature or logg values from the KIC.

Bonomo \& Lanza (2012) analyze the active planet-hosting star Kepler-17 (KIC 10619192). They detect a rotation period of $12.01 \mathrm{~d}$ and the existence of two active longitudes separated by approx. $180^{\circ}$ from each other. They found evidence for solar-like DR but were not able to give precise estimates for the horizontal shear since the active regions evolve on timescales similar to the rotation rate. We detect an alias period of $6.05 \mathrm{~d}$ due to the two active longitudes. Again, we find no second period within $30 \%$ of $P_{1}$ although the second and third strongest period we found are 10.85 and 12.28 days respectively, in agreement with the stated rapid spot evolution. Recently, Roettenbacher et al. (2013) find a period of 3.47 days for the Kepler target KIC 5110407, and evidence for DR using light curve inversion. We find $P_{1}=3.61$ and $P_{2}=3.42$ days yielding $\alpha=0.053$ which is consistent with their differential rotation coefficient $k=0.053 \pm 0.014$ for an inclination of $i=45^{\circ}$ in their model.

Several open clusters in the Kepler field have been studied. Meibom et al. (2011) measure rotation periods for stars in the open cluster NGC 6811, supplying evidence for rotational braking (comp. Fig. 8.2). Further measurements have been done for the open clusters NGC 6866 (Balona et al. 2013a) and NGC 6819 (Balona et al. 2013b) showing similar rotation behavior. In the next chapter we compare our results to theoretical predictions. 


\section{Comparison to theory}

Solar DR has theoretically been studied for a long time. Kitchatinov \& Rüdiger (1999) compute mean-field models for late-type (G2 and K5) stars. They find that the relative shear $\alpha$ increases with rotation period. They also show that $\alpha$ increases towards cooler stars. The general trends are in agreement with our findings, although their range of $\alpha$ values lies above our values.

Küker \& Rüdiger (2005a) compute models for an F8 star and find weak dependence on rotation period, which is confirmed by later studies for F, G and K stars (Küker \& Rüdiger 2005b) showing that the dependence on temperature is much stronger. The latter result holds for different main sequence star models (Küker \& Rüdiger 2007). They find that above a temperature of $5800 \mathrm{~K}$ the fit from Barnes et al. (2005) fits the model data reasonably well whereas below $5800 \mathrm{~K}$ the data lies far off the fit. Recent studies (Küker \& Rüdiger 2011) have shown that the temperature dependence of $\Delta \Omega$ cannot be represented by one single power law over the whole temperature range from 3800-6700 K. Fig. 2 in Küker \& Rüdiger (2011) shows that $\Delta \Omega$ only slightly increases with temperature for stars cooler than $\approx 5800 \mathrm{~K}$ consistent with our measurements (Fig. 8.8). For stars hotter than $\approx 6200 \mathrm{~K}$ they find that $\Delta \Omega$ shows even stronger dependence on temperature than predicted by Barnes et al. (2005). This is the same temperature region where our measurements start to show a different behavior (comp. Fig. 8.8).

The weak dependence of $\Delta \Omega$ on rotation period is confirmed for different solar mass models. Hotta \& Yokoyama (2011) model DR of rapidly rotating solar-type stars and find that DR approaches the Taylor-Proudman state, i.e. that $\Delta \Omega / \Omega$ decreases with angular velocity as long as the rotation rate is above the solar value.

Direct numerical simulations show that models with low Rossby numbers (i.e. fast rotating stars) generate strong dipolar magnetic fields (Schrinner et al. 2012). These fields amplify Lorentz forces able to suppress Coriolis forces, and hence can effectively suppress DR (Gastine et al. 2012). This result from theoretical models agrees well with the trend we found that $\alpha$ decreases towards shorter rotation periods.

The dipolarity of the magnetic field strongly depends on the length scale of convection. As the depth of the convection zone decreases the dipolarity breaks down rendering the rotation non-uniform (Schrinner et al. 2012). This effect could explain the strong increase of $\Delta \Omega$ around $T_{\text {eff }}>6000 \mathrm{~K}$. Browning (2011) provides an explanation for the shallow increase of $\alpha$ towards cooler stars. M dwarfs exhibit low luminosities, and therefore low convective velocities. Thus, they strongly depend on the rotation rate even at solar rotation rates. Magnetic fields cannot quench DR effectively so they exhibit strong solar-like DR.

We have seen that theoretical models partly explain our results but a theory of DR consistent with previous and recent observations is still pending. I hope that this work will help theoreticians to constrain their parameter space, and to understand the outcome of the model being used. Combining theoretical and observational effort will hopefully provide insight into the nature of different dynamo mechanisms in the near future. 


\section{Summary \& Conclusions}

We have run a large Monte-Carlo simulation of differentially rotating, spotted stars covering a significant fraction of the parameter space. The resulting light curves have been analyzed with the Lomb-Scargle periodogram in a prewhitening approach. The returned periods have been used for a global sine fit to the data. The major goal was to see under which stellar conditions and upon what accuracy DR can be detected.

For all stars with at least one visible spot we calculate a rotation period. This period $P 1_{\text {out }}$ is the most significant one in the data, and could be detected for $96.2 \%$ of all light curves. A second period close to $P 1_{\text {out }}$ has been attributed to a second spot found in $64.2 \%$ of all cases. The latitudinal shear of the two spots associated to the above periods has been calculated. We found that the shear of the two spots $\alpha_{\text {in }}$ was on average $3.2 \%$ lower than $\alpha_{\text {out }}$. Furthermore, comparing $\alpha_{\text {out }}$ to the total equator-to-pole shear we find that $\alpha$ has been underestimated by $8.8 \%$. Especially, we found that the detection of DR is challenging for stars with an equator-topole shear of less than $6 \%$ which usually yields large errors.

In our model each light curve is composed by a fixed number of spots rotating at defined latitudes. On the sun the situation is different. Spots vanish or are created while rotation takes place. During the 11-year activity cycle they migrate towards the equator. So far, our program does not account for meridional drifts, and spot lifetimes have recently been implemented but have not been tested. The least known model parameter is the number of spots on the surface and their associated size. Alternative to our approach, one could also use more spots with smaller radii keeping the spot filling factor constant, i.e. the fraction of the surface covered with spots. But this also increases the number of available spot periods. Comparing them to the outcome of our analysis bears the risk of choosing a spot that is not responsible for the detected signal.

Our method is limited to a relative DR of $\alpha<0.5$. Allowing for $\alpha \geq 0.5$ in the model would lead to confusion between real spot periods and aliases of faster rotating spots with $P 1 \leq 1 / 2 P 2$. This is a general problem of DR determination from photometric data.

Our lower limit in relation (3.1) accounts for the frequency resolution in the periodograms. This limit is only relevant to keep reasonable computation time and could in principle be discarded in contrast to the upper limit. Observations of DR cover a wide range of $\alpha$ values. The Doppler Imaging technique is particularly sensitive to small DR limited by the spot lifetimes, $\alpha \lesssim 0.01$, although there are measurements (Donati et al. 2003) which yield $\alpha \approx 0.05$ for LQ Hya, and new measurements (Marsden et al. 2011) who find values between $0.005 \lesssim \alpha \lesssim 0.14$. The Fourier transform method (e.g. Reiners \& Schmitt (2003)) is sensitive to $\alpha>0.1$ and has been used to determine surface shears as large as $50 \%$ for some A-F stars. We conclude that our results confirm the possibility to reliably detect DR from photometric data using a fast tool with relatively simple mathematical assumptions.

We have applied our method to the active fraction of Kepler Q3 data to search for DR in high precision empirical data. We have measured rotation periods of 24.124 Kepler stars providing evidence for DR in 18.619 stars. Our measurements for the rotation period are in good agreement with previous results. Moreover they are consistent with the theory of magnetic braking.

Our measurements provide a comprehensive database of stellar differential rotation. For the first 
time, we can explore a well-defined parameter range in a statistically significant sample. We found that the relative shear $\alpha$ increases towards longer periods and slightly increases towards cooler stars. For periods greater than 2 days the absolute shear $\Delta \Omega$ shows only weak dependence on rotation period. In contrast to other observations $\Delta \Omega$ shows a shallow increase for temperatures from $3500-6000 \mathrm{~K}$, and a steep increase above $6000 \mathrm{~K}$. Both the dependence on rotation period and temperature are in good agreement with recent theoretical models. Furthermore, we cannot find any other reasonable explanation for the trends we found.

We interpret the existence of a second period as DR. Although our method is not able to distinguish between DR and spot evolution we are confident that most of our measurements reflect the stellar surface shear because our results resemble previous measurements and recent theoretical models. This is the first time that DR has been measured for such a large number of stars. Some rotation periods for individual targets may be wrong but the general trends we found are consistent with theory and observations. For the future this analysis will be applied to more Kepler data to verify the rotation periods, esp. the DR, and to learn about spot lifetimes. Kepler is a natural source of answers to these questions. 


\section{Outlook}

The main question that could not be answered in this work is how one can distinguish between DR and spot evolution. Analytical spot models usually account for DR and spot lifetimes in the parameter space. On the sun active regions evolve during one rotation cycle but recent results have shown that rotation periods (and hence active regions) can be very stable over time spans up to 2 years (Nielsen et al. 2013). Spot evolution has been implemented in MODSTAR by coupling the spot radii to the number of rotation steps, i.e. in each rotation step the spot radii increase or decrease by a defined amount. Tests show that in very simple situations, e.g. for a two spot model, the periods returned by our method are different which means that - in principle - one could differentiate between these effects. For the future we plan to develop simple stellar models to understand the outcome of our method. Only then spot lifetimes can be considered as further parameter in another Monte-Carlo simulation.

Furthermore, we plan to apply our method to the whole time coverage of Kepler observations. In this way we hope to constrain our periods, and to achieve estimates of spot lifetimes. Up to now Kepler has provided data from 02 May 2009 - 11 January 2013 (start of Q0 until end of Q15). Unfortunately, the telescope had to stop science operations so far since two of the four gyroscopes are out of order. Although the future of the telescope is uncertain Kepler has provided a huge amount of high quality data that will engage scientists for a long time. 



\section{Bibliography}

Aerts, C., Christensen-Dalsgaard, J., \& Kurtz, D. W. 2010, Asteroseismology (Springer Netherlands)

Affer, L., Micela, G., Favata, F., \& Flaccomio, E. 2012, MNRAS, 424, 11

Ammler-von Eiff, M. \& Reiners, A. 2012, A\&A, 542, A116

Baliunas, S., Sokoloff, D., \& Soon, W. 1996, ApJ, 457, L99

Baliunas, S. L., Hartmann, L., Noyes, R. W., et al. 1983, ApJ, 275, 752

Balona, L. A., Guzik, J. A., Uytterhoeven, K., et al. 2011a, MNRAS, 415, 3531

Balona, L. A., Guzik, J. A., Uytterhoeven, K., et al. 2011b, MNRAS, 415, 3531

Balona, L. A., Joshi, S., Joshi, Y. C., \& Sagar, R. 2013a, MNRAS, 429, 1466

Balona, L. A., Medupe, T., Abedigamba, O. P., et al. 2013b, MNRAS

Baraffe, I., Chabrier, G., Allard, F., \& Hauschildt, P. H. 1998, A\&A, 337, 403

Barnes, J. R., Collier Cameron, A., Donati, J.-F., et al. 2005, MNRAS, 357, L1

Barnes, S. A. 2003, ApJ, 586, 464

Barnes, S. A. 2007, ApJ, 669, 1167

Basri, G., Walkowicz, L. M., Batalha, N., et al. 2011, AJ, 141, 20

Basri, G., Walkowicz, L. M., Batalha, N., et al. 2010, ApJ, 713, L155

Berdyugina, S. V. 2005, Living Reviews in Solar Physics, 2, 8

Bonomo, A. S. \& Lanza, A. F. 2012, A\&A, 547, A37

Browning, M. K. 2011, in IAU Symposium, Vol. 271, IAU Symposium, ed. N. H. Brummell, A. S. Brun, M. S. Miesch, \& Y. Ponty, 69-77

Budding, E. 1977, Ap\&SS, 48, 207

Claret, A. 2000, A\&A, 363, 1081

Collier Cameron, A. 2007, Astronomische Nachrichten, 328, 1030

Collier Cameron, A., Donati, J.-F., \& Semel, M. 2002, MNRAS, 330, 699

Covey, K. R., Agüeros, M. A., Lemonias, J. J., et al. 2011, in Astronomical Society of the Pacific Conference Series, Vol. 448, 16th Cambridge Workshop on Cool Stars, Stellar Systems, and the Sun, ed. C. Johns-Krull, M. K. Browning, \& A. A. West, 269

Croll, B., Walker, G. A. H., Kuschnig, R., et al. 2006, ApJ, 648, 607 
Debosscher, J., Blomme, J., Aerts, C., \& De Ridder, J. 2011, A\&A, 529, A89

Donahue, R. A., Saar, S. H., \& Baliunas, S. L. 1996, ApJ, 466, 384

Donati, J.-F. \& Collier Cameron, A. 1997, MNRAS, 291, 1

Donati, J.-F., Collier Cameron, A., \& Petit, P. 2003, MNRAS, 345, 1187

Dorren, J. D. 1987, ApJ, 320, 756

Frasca, A., Fröhlich, H.-E., Bonanno, A., et al. 2011, A\&A, 532, A81

Fröhlich, H.-E., Frasca, A., Catanzaro, G., et al. 2012, A\&A, 543, A146

Fröhlich, H.-E., Küker, M., Hatzes, A. P., \& Strassmeier, K. G. 2009, A\&A, 506, 263

Gastine, T., Duarte, L., \& Wicht, J. 2012, A\&A, 546, A19

Gilliland, R. L. \& Fisher, R. 1985, PASP, 97, 285

Gizon, L. \& Solanki, S. K. 2004, Sol. Phys., 220, 169

Hall, D. S. 1991, in Lecture Notes in Physics, Berlin Springer Verlag, Vol. 380, IAU Colloq. 130: The Sun and Cool Stars. Activity, Magnetism, Dynamos, ed. I. Tuominen, D. Moss, \& G. Rüdiger, 353

Hotta, H. \& Yokoyama, T. 2011, ApJ, 740, 12

Huber, K. F., Czesla, S., Wolter, U., \& Schmitt, J. H. M. M. 2010, A\&A, 514, A39

Irwin, J., Berta, Z. K., Burke, C. J., et al. 2011, ApJ, 727, 56

Jester, S., Schneider, D. P., Richards, G. T., et al. 2005, AJ, 130, 873

Kipping, D. M. 2012, MNRAS, 427, 2487

Kiraga, M. \& Stepien, K. 2007, Acta Astron., 57, 149

Kitchatinov, L. L. 2005, Physics Uspekhi, 48, 449

Kitchatinov, L. L. \& Rüdiger, G. 1999, A\&A, 344, 911

Küker, M. \& Rüdiger, G. 2005a, A\&A, 433, 1023

Küker, M. \& Rüdiger, G. 2005b, Astronomische Nachrichten, 326, 265

Küker, M. \& Rüdiger, G. 2007, Astronomische Nachrichten, 328, 1050

Küker, M. \& Rüdiger, G. 2011, Astronomische Nachrichten, 332, 933

Lanza, A. F., Rodono, M., \& Zappala, R. A. 1993, A\&A, 269, 351

Marsden, S. C., Jardine, M. M., Ramírez Vélez, J. C., et al. 2011, MNRAS, 413, 1939

McQuillan, A., Aigrain, S., \& Mazeh, T. 2013, ArXiv e-prints

Meibom, S., Barnes, S. A., Latham, D. W., et al. 2011, ApJ, 733, L9

Morin, J., Donati, J.-F., Forveille, T., et al. 2008, MNRAS, 384, 77 
Morin, J., Donati, J.-F., Petit, P., et al. 2010, MNRAS, 407, 2269

Nielsen, M. B., Gizon, L., Schunker, H., \& Karoff, C. 2013, ArXiv e-prints

Reed, B. C. 1998, JRASC, 92, 36

Reiners, A. 2006, A\&A, 446, 267

Reiners, A. \& Mohanty, S. 2012, ApJ, 746, 43

Reiners, A. \& Schmitt, J. H. M. M. 2002, A\&A, 384, 155

Reiners, A. \& Schmitt, J. H. M. M. 2003, A\&A, 398, 647

Roettenbacher, R. M., Monnier, J. D., Harmon, R. O., Barclay, T., \& Still, M. 2013, ArXiv e-prints

Savanov, I. S. 2011a, Astronomy Reports, 55, 801

Savanov, I. S. 2011b, Astronomy Reports, 55, 341

Savanov, I. S. \& Dmitrienko, E. S. 2011, Astronomy Reports, 55, 890

Savanov, I. S. \& Dmitrienko, E. S. 2012, Astronomy Reports, 56, 116

Schrinner, M., Petitdemange, L., \& Dormy, E. 2012, ApJ, 752, 121

Skumanich, A. 1972, ApJ, 171, 565

Strassmeier, K. G. 2009, A\&A Rev., 17, 251

Uytterhoeven, K., Moya, A., Grigahcène, A., et al. 2011, A\&A, 534, A125

Walkowicz, L. M., Basri, G. S., \& Valenti, J. A. 2012, ArXiv e-prints

Zechmeister, M. \& Kürster, M. 2009, A\&A, 496, 577 



\section{Curriculum Vitae}

Timo Reinhold

Planckstr. 21

37073 Göttingen

Geburtstag: 21.09.1983

Geburtsort: Bremerhaven

Staatsangehörigkeit: deutsch

\section{Ausbildung}

\section{Universität}

Juni 2010 - voraus. August 2013: Doktorand am Institut für Astrophysik, Georg-August-Universität Göttingen

Juni 2009: Diplom Physik, Georg-August-Universität Göttingen

April 2006: Vordiplom Physik, Georg-August-Universität Göttingen

Oktober 2003 - Juni 2009: Diplomstudiengang Physik, Georg-August-Universität Göttingen

\section{Schule}

Juni 2003: Abitur an der Oberstufe Carl von Ossietzky, Bremerhaven

2000 - 2003: Oberstufe Carl von Ossietzky, Bremerhaven

1996 - 2000: Gymnasium Loxstedt

1994 - 1996: Orientierungsstufe Loxstedt

1990 - 1994: Heinrich-Luden Grundschule Loxstedt 

